NASA/CR-2000-210556

ICASE Report No. 2000-42

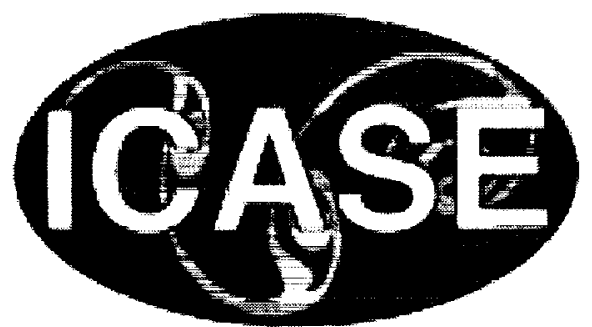

\title{
On the Modeling of Shells in Multibody Dynamics
}

Olivier A. Bauchau and Jou-Young Choi

Georgia Institute of Technology, Atlanta, Georgia

Carlo L. Bottasso

Politecnico di Milano, Milano, Italy 


\section{The NASA STI Program Office ... in Profile}

Since its founding, NASA has been dedicated to the advancement of aeronautics and space science. The NASA Scientific and Technical Information (STI) Program Office plays a key part in helping NASA maintain this important role.

The NASA STI Program Office is operated by Langley Research Center, the lead center for NASA's scientific and technical information. The NASA STI Program Office provides access to the NASA STI Database, the largest collection of aeronautical and space science STI in the world. The Program Office is also NASA's institutional mechanism for disseminating the results of its research and development activities. These results are published by NASA in the NASA STI Report Series, which includes the following report types:

- TECHNICAL PUBLICATION. Reports of completed research or a major significant phase of research that present the results of NASA programs and include extensive data or theoretical analysis. Includes compilations of significant scientific and technical data and information deemed to be of continuing reference value. NASA's counterpart of peer-reviewed formal professional papers, but having less stringent limitations on manuscript length and extent of graphic presentations.

- TECHNICAL MEMORANDUM. Scientific and technical findings that are preliminary or of specialized interest, e.g., quick release reports, working papers, and bibliographies that contain minimal annotation. Does not contain extensive analysis.

- CONTRACTOR REPORT. Scientific and technical findings by NASA-sponsored contractors and grantees.
- CONFERENCE PUBLICATIONS. Collected papers from scientific and technical conferences, symposia, seminars, or other meetings sponsored or cosponsored by NASA.

- SPECIAL PUBLICATION. Scientific, technical, or historical information from NASA programs, projects, and missions, often concemed with subjects having substantial public interest.

- TECHNICAL TRANSLATION. Englishlanguage translations of foreign scientific and technical material pertinent to NASA's mission.

Specialized services that complement the STI Program Office's diverse offerings include creating custom thesauri, building customized data bases, organizing and publishing research results ... even providing videos.

For more information about the NASA STI Program Office, see the following:

- Access the NASA STI Program Home Page at http://www.sti.nasa.gov

- Email your question via the Internet to help@sti.nasa.gov

- Fax your question to the NASA STI Help Desk at (301) 621-0134

- Telephone the NASA STI Help Desk at (301) 621-0390

- Write to: NASA STI Help Desk NASA Center for AeroSpace Information 7121 Standard Drive Hanover, MD 21076-1320 
NASA/CR-2000-210556

ICASE Report No. 2000-42

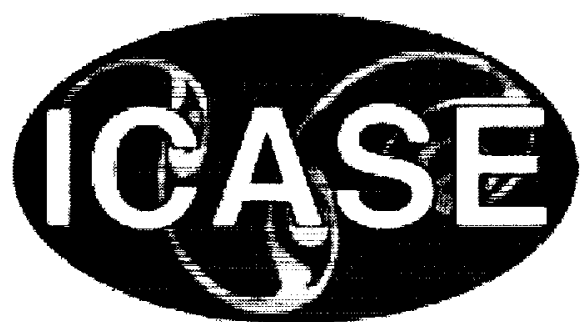

\section{On the Modeling of Shells in Multibody Dynamics}

Olivier A. Bauchau and Jou-Young Choi

Georgia Institute of Technology, Atlanta, Georgia

Carlo L. Bottasso

Politecnico di Milano, Milano, Italy

ICASE

NASA Langley Research Center

Hampton, Virginia

Operated by Universities Space Research Association

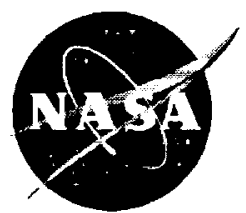

National Aeronautics and

Space Administration

Langley Research Center

Hampton, Virginia 23681-2199 
Available from the following:

NASA Center for AcroSpace Information (CASI)

7121 Standard Drive

Hanover, MD 21076-1320

(301) $621-0390$

National Technical Information Service (NTIS)

5285 Port Royal Road

Springfield, VA 22161-2171

(703) 487-4650 


\title{
ON THE MODELING OF SHELLS IN MULTIBODY DYNAMICS
}

\author{
OINIER A. BAUCHAU*, JOI YOUNG CHOI ${ }^{\dagger}$, AND CARI.O I. BOTTASSO
}

\begin{abstract}
Energy preserving/decaying schemes are presented for the simulation of the nonlinear multibody systems involving shell components. The proposed schemes are designed to meet four specific requirements: unconditional nonlinear stability of the scheme, a rigorous treatment of both geometric and material nonlinearities, exact satisfaction of the constraints, and the presence of high frequency numerical dissipation. The kinematic nonlinearities associated with arbitrarily large displacements and rotations of shells are treated in a rigorous manner, and the material nonlinearities can be handled when the constitutive laws stem from the existence of a strain energy density function. The efficiency and robustness of the proposed approach is illustrated with specific numerical examples that also demonstrate the need for integration schemes possessing high frequency numerical dissipation.
\end{abstract}

Key words. multibody dynamics, geometrically exact shell, time integration, energy decaying scheme

Subject classification. Applied and Numerical Mathematics

1. Introduction and Motivation. This work is concerned with the numerical simulation of geometrically exact shell models within the context of multibody system dynamics. While the partial differential equations that govern shell problems are well known and presented in numerous textbooks, their numerical treatment is still the subject of active research. Indeed, numerical analysis tools for partial differential equations have significantly changed in recent years. In the past, general purpose discretization methods were developed, with emphasis on robustness, performance, and accuracy. These methods aimed at solving vast classes of problems such as ordinary differential equations, differential/algebraic equations, or hyperbolic conservation laws.

This approach is now changing. The differential cquations that govern many problems in mathematical physics possess qualitative and structural characteristics that can be determined by studying their geometry. Classical examples of such characteristics are the invariants associated with Hamiltonian systems, the symplectic structure of the governing equations, or symmetries and attractors. There is increasing evidence that numerical methods that correctly recover the qualitative features of the underlying differential equations are often endowed with superior computational performance, greater robustness and improved accuracy.

This new paradigm has resulted in the development of a new mathematical discipline, called geometric integration, whose aim can be summarized as: "the purpose of computing is insight, not numbers", to quote Hamming [17]. Geometric integration theory is now becoming a bridge that links the work of pure, applied and computational mathematicians, and it is usually expressed using the terminology and formalism of differential geometry and Lie group theory.

In reality, engineers have used geonetric integration for a number years. Simo and his co-workers were among the first to develop special integration procedures for nonlinear structural dynamics. They analyzed

\footnotetext{
*Georgia Institute of Technology, School of Aerospace Engineering, Atlanta, GA, USA (email address: Olivier.Bauchau Oaerospace.gatech.edu).

tGeorgia Institute of Terhnology, School of Aerospace Fngineering, Atlanta, GA, USA.

¥Dipartimento di Ingegneria Aerospaziale, Politecnico di Milano, Via Ja Masa 34, 20158 Milann, Italy (email address: Carlo. Bottasso opolimi.it). This research was supported by the National Aeronautics and Space Administration under NASA Contract No. NAS1-97046 while the third author was in residence at ICASE, NASA Iangley Research Center, Hampton, VA 23681-2199.
} 
the problem of the dynamics of rigid bodies [24], nonlinear elasto-dynamics [21], geometrically exact shells [22] and geometrically exact beams [23]. In all cases, the idea was to design algorithms that ensure the discrete preservation of the total mechanical energy of the system, therefore obtaining unconditionally stable schemes in the nonlinear regime. These energy methods, sometimes equipped with additional conservation properties, such as the conservation of momenta, are becoming increasingly popular in multibody dynamirs, paralleling, although unknowingly, the mathematical developments of geometric integration theory.

However, increasing evidence points toward the fact that geometric integration is not sufficient, per se, to obtain robust integration schemes. While these schemes perform well for problems with a small number of degrees of freedom or those featuring a "smooth" dynamic response, they tend to be quite unsatisfactory when applied to the complex simulations encountered in many engineering applications [6]. In fact, the predicted time histories of internal forces and velocities can present a significant high frequency content. Furthermore, the presence of these high frequency oscillations hinders the convergence process for the solution of the nonlinear equations of motion. The selection of a smaller time step does not necessarily help, as a smaller time step allows even higher frequency oscillations to be present in the response. These oscillations are particularly violent in multibody dynamics simulations because these systems are rather stiff due to the presence of numerous algebraic constraints, while the nonlinearities of the system provide a mechanism to transfer energy from the low to the high frequency modes. These difficulties can easily go unnoticed in many test cases, but will create major problems for large scale simulations. Consequently, the presence of high frequency numerical dissipation appears to be an indispensable feature of robust time integrators for multibody systems. Such feature can be added to schemes developed within the framework of geometric integrators, as it will be shown in this paper. High frequency numerical dissipation does not alter the physics of the problem, since the high frequency content of the response filtered out by the algorithm is itself an artifact of the spatial discretization process and contains no information about the physical behavior of the system. The need for high frequency dissipation in large finite element models has been recognized for many years in various disciplines, from structural dynamics to fluid mechanics. The most widely used time integrators for finite element analysis are high frequency dissipative $[19,18]$.

This paper focuses on the development of a geometric integrator for shell structures that preserves important qualitative features of the underlying equations, and is equipped with high frequency numerical dissipation. The goal of the work is to obtain schemes presenting improved robustness and reliability over standard "black-box" integrators. In order to achieve this goal, the specific features of the equations governing nonlinear flexible multibody systems with shells are reviewed.

First, the governing equations are characterized by linear and rotational tensorial fields describing kinematic (displacements, velocities) and co-kinematic (forces, momenta) quantities. In shells, the rotational field describes the evolution of unit director, and is therefore a special family of two-parameter rotations. Second, the equations are nonlinear because of large displacements and finite rotations (geometric nonlinearities), and possibly because of nonlinear constitutive laws (material nonlinearities). Third, the presence of joints imposes different types of kinematic constraints between the various bodies of the system. In this work, the Lagrange multipliers technique is used to enforce the constraints, giving the governing equations a differential/algebraic nature. Fourth, the equations of motion imply the preservation of a number of dynamic invariants, in particular the total mechanical energy, and the total linear and angular momenta.

Since discrete preservation of energy leads to unconditional nonlinear stability; preservation of this invariant is a central focus of this paper. The proposed geometric integration procedure is therefore designed to satisfy specific requirements. First, a discretization process is developed that preserves the total mechanical 
energy of the system at the discrete solution level, as well as the total linear and angular momenta. This process is independent of the spatial discretization procedure that is left arbitrary. In the present implementation, the finite element method is used, and the mixed interpolation of tensorial components $[1,2,16]$ is implemented to avoid the shear locking problem. Next, the reaction forces associated with the holonomic and non-holonomic constraints imposed on the system are discretized in a manner that guarantees the satisfaction of the nonlinear constraint manifold, i.e. the constraint condition will not drift. At the same time, the discretization implies the vanishing of the work performed by the forces of constraint at the discrete solution level. Consequently, the discrete energy conservation laws proved for the flexible members of the system are not upset by the introduction of the constraints. The resulting Energy Preserving (EP) scheme is a geometric integrator for multibody systems with shells that provides nonlinear unconditional stability. However, it clearly lacks the indispensable high frequency numerical dissipation required to tackle realistic engineering problems.

Using a simple procedure $[3,4]$ based on the EP scheme, it is possible to derive a new discretization that implies a discrete energy deray statement. In the resulting Energy Decaying (ED) scheme, the system no longer evolves on the constant energy level set, but is allowed to drift away from it in a controlled manner. This concept seems to be new in geometric integration theory, and provides a procedure to obtain nonlinear unconditional stability (from the bound on the encrgy), together with a mechanism for removing the undesired high frequencies. The discretization process for the forces of constraint is left unchanged: the work they perform vanishes exactly, while the system evolves on the constraint manifold without drifts. Therefore ED schemes satisfy all the requirements set forth earlier.

Related ED schemes for various problems in multibody dynamics were proposed in the literature. Finite difference schemes were presented in $[8,9,5,13]$. Time discontinuous Galerkin approximations of the equations of motion written in the symmetric hyperbolic form are used in [7,3]. In [14], ED schemes are cast in the form of Runge-Kutta schemes and related to the basic concepts of geometric integration theory. The analysis in ref. [4] shows that slightly different EP and ED discretizations can be developed, usually through different treatments or parameterizations of finite rotations. Some discretizations might also present additional conservation properties. For instance, some EP and ED schemes also imply the conservation of momenta, or are geometrically invariant $[10,15,4]$. These additional features are easily obtained by recasting the field equations in fixed pole form [11], a procedure that brings back again the link with Lie groups through the use of exponentials and of Cayley's transform.

The paper is laid out as follows. The equations of motion for shells are presented in section 2. The energy preserving scheme presented in section 3 is then generalized to an energy decaying scheme in section 4 . A special element, the shell revolute joint, used to connect shells to other components of a multibody system, is developed in section 5. Finally, numerical examples are presented in section 6 .

\section{Formulation of the Equations of Motion.}

2.1. Shell Kinematics. Consider a shell of thickness $h$ and reference surface area $\Omega$, as depicted in fig. 2.1. An inertial frame of reference $\mathcal{S}$ consisting of three mutually orthogonal unit vectors $\mathbf{i}_{1}, \mathbf{i}_{2}, \mathbf{i}_{3}$ is used. Let $\underline{r}_{0}$ be the position vector of an arbitrary point on the reference surface of the shell, and let $\zeta$ be the material coordinate along $\underline{n}$, the normal to the reference surface. The position vector $\underline{r}$ of an arbitrary point on the shell in its reference configuration is then

$$
\underline{r}\left(\xi^{1}, \xi^{2}, \zeta\right)=\underline{r}_{0}\left(\xi^{1}, \xi^{2}\right)+\zeta \underline{n}\left(\xi^{1}, \xi^{2}\right)
$$




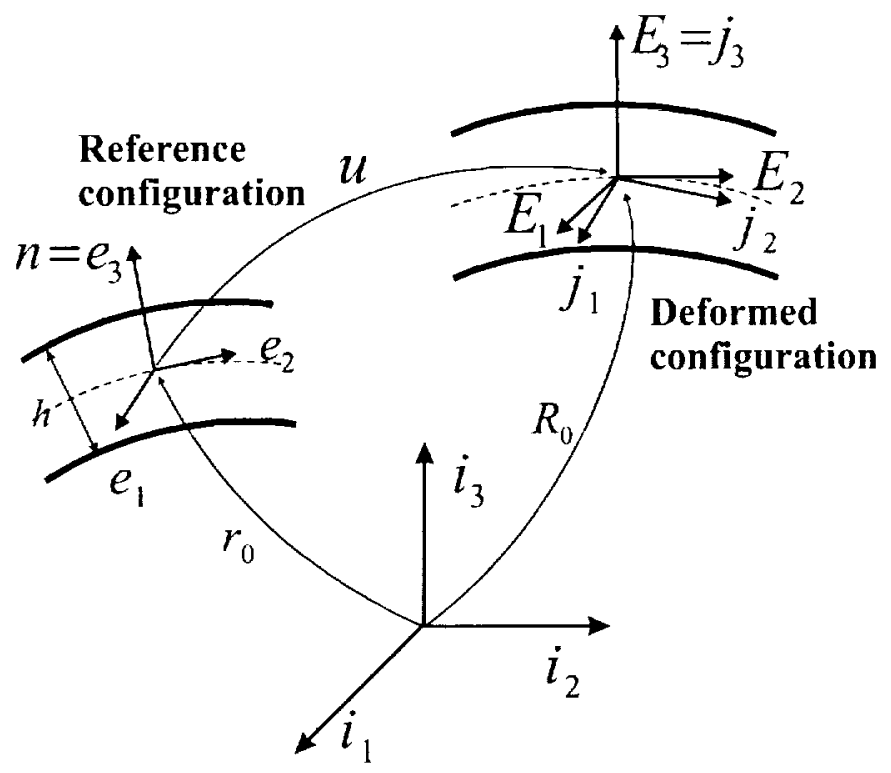

FIG. 2.1. Configuration of the shell in the reference and deformed configurations.

where $\xi^{1}$ and $\xi^{2}$ are the material coordinates used to represent the shell reference surface. The coordinates $\xi^{1}, \xi^{2}$ and $\zeta$ form a set of curvilinear coordinates that are a natural choice to represent the shell geometry. The coordinates $\xi^{1}$ and $\xi^{2}$ are assumed to be lines of curvatures of the shell reference surface. The base vectors are then

$$
g=\left[\underline{g}_{1}, \underline{g}_{2}, \underline{g}_{3}\right]=\left[\underline{r}_{1}, \underline{r}_{2}, \frac{\partial \underline{r}}{\partial \zeta}\right]=\left[\left(1-\frac{\zeta}{R_{1}}\right) \underline{a}_{1},\left(1-\frac{\zeta}{R_{2}}\right) \underline{a}_{2}, \underline{n}\right],
$$

where $R_{1}$ and $R_{2}$ are the principal radii of curvature, $\underline{a}_{\alpha}=\underline{r}_{0, \alpha}$, and the notation $(\bullet)_{, \alpha}$ is used to denote a derivative with respect to $\xi^{\curvearrowright}$. It is convenient to introduce a set of three mutually orthogonal unit vectors at the shell reference surface (i.e. at $\zeta=0$ )

$$
\underline{e}_{1}=\frac{\underline{a}_{1}}{\sqrt{a_{11}}} ; \quad \underline{e}_{2}=\frac{\underline{a}_{2}}{\sqrt{a_{22}}} ; \quad \underline{e}_{3}=\underline{n},
$$

where $a_{\alpha \alpha}=\underline{a}_{\alpha} \cdot \underline{a}_{\alpha}$.

According to the classical inextensible director model, it is assumed that the material line initially normal to the reference surface of the shell remains a straight line and suffers no extension. Therefore, the position vector of a material point of the shell can be written as

$$
\underline{R}\left(\xi^{1}, \xi^{2}, \zeta\right)=\underline{r}_{0}\left(\xi^{1}, \xi^{2}\right)+\underline{u}\left(\xi^{1}, \xi^{2}\right)+\zeta \underline{E}_{3}\left(\xi^{1}, \xi^{2}\right),
$$

where $\underline{u}\left(\xi^{1}, \xi^{2}\right)$ is the reference surface displacement vector. The base vectors at the shell reference surface in the deformed configuration are

$$
G=\left[\underline{G}_{1}, \underline{G}_{2}, \underline{G}_{3}\right]=\left[\underline{R}_{1}, \underline{R}_{, 2}, \frac{\partial \underline{R}}{\partial \zeta}\right] .
$$

Introduring the position vector, eq. (2.4), then yields

$$
\hat{G}=\left[\frac{\underline{G}_{1}}{\sqrt{a_{11}}}, \frac{\underline{G}_{2}}{\sqrt{a_{22}}}, \underline{G}_{3}\right]=E+\zeta H
$$


where

$$
F=\left[\underline{E}_{1}, \underline{E}_{2}, \underline{E}_{3}\right]=\left[\underline{e}_{1}+\frac{\underline{u}_{, 1}}{\sqrt{a_{11}}}, \underline{e}_{2}+\frac{\underline{u}_{, 2}}{\sqrt{a_{22}}}, \underline{E}_{3}\right] ; \quad H=\left[\frac{\underline{E}_{3,1}}{\sqrt{a_{11}}}, \frac{\underline{F}_{3,2}}{\sqrt{a_{22}}}, 0\right] .
$$

Note that $\underline{E}_{3}\left(\xi^{1}, \xi^{2}\right)$ is a unit vector, whereas $\underline{E}_{1}$ and $\underline{E}_{2}$ are not unit vectors, nor are they orthogonal to $\underline{E}_{3}$, as axial and transverse shearing strains develop during deformation.

2.2. Equations of Motion. The Green-Lagrange strain tensor $e$ is defined as

$$
e=\frac{1}{2}\left(G^{T} G-g^{T} g\right)
$$

The strain tensor $e$ is defined in the curvilinear coordinate system defined by coordinates $\xi^{1}, \xi^{2}$ and $\zeta$. However, it is more convenient to work with strain tensor $e$ defined in the locally rectangular system defined by triad $\underline{e}_{1}, \underline{e}_{2}, \underline{e}_{3}$, see eqs. (2.3). For shallow shells (i.e. $\zeta / R_{1} \ll 1$ and $\left.\zeta / R_{2} \ll 1\right)$ undergoing large displacements and rotations but small strains (all strain components are assumed to be small compared to unity), the strain-displaccment relationships can be written as

$$
e=\frac{1}{2}\left[E^{T} E-I+\zeta\left(E^{T} H+H^{T} E+\kappa\right)\right]
$$

where

$$
\kappa=\left[\begin{array}{ccc}
1 / R_{1} & 0 & 0 \\
0 & 1 / R_{2} & 0 \\
0 & 0 & 0
\end{array}\right]
$$

It is clear that the strains can be expressed in terms of five parameters: the three components of the displacement field $\underline{u}$ (through $\underline{E}_{1}$ and $\underline{E}_{2}$ ) and the two parameters defining the orientation of the unit director $\underline{E}_{3}$. Virtual changes in the strain energy of the structure are given by

$$
\delta V=\int_{\Omega} \int_{h} \delta \bar{V} \mathrm{~d} \zeta \mathrm{d} \Omega=\int_{\Omega} \int_{h} \delta e \cdot \tau \mathrm{d} \zeta \mathrm{d} \Omega
$$

where $\delta \bar{V}$ is the virtual strain energy density, and $\tau$ the second Piola-Kirchhoff stress tensor. Introducing the strains, eq. (2.9), and taking into account the symmetry of the stress tensor then yields

$$
\delta \bar{V}=\delta E \cdot(E+\zeta H) \tau+\delta H \cdot \zeta E \tau
$$

The existence of a strain energy density function $\bar{V}$ is postulated here, hence the constitutive laws are of the form $\tau=\partial \bar{V} / \partial e$.

The velocity vector of material point $P$ of the shell is obtained by differentiating the position vector, eq. (2.4), with respect to time, to find $\underline{v}=\underline{\dot{u}}+\zeta \underline{\dot{E}}_{3}$. The kinetic energy of the system is now

$$
K=\int_{\Omega} \int_{h} \bar{K} \mathrm{~d} \zeta \mathrm{d} \Omega=\frac{1}{2} \int_{\Omega} \int_{h} \rho \underline{v} \cdot \underline{v} \mathrm{~d} \zeta \mathrm{d} \Omega
$$

where $\bar{K}$ is the kinetic energy density. Introducing the velocity vector then yields

$$
\bar{K}=\frac{1}{2} \rho\left(\underline{\dot{u}}+\zeta \underline{\dot{E}}_{3}\right) \cdot\left(\underline{i}+\zeta \underline{E}_{3}\right)
$$


Hamilton's Principle now writes

$$
\begin{aligned}
\int_{t_{1}}^{t_{f}} \int_{\Omega} \int_{h}(\delta \bar{K}-\delta \bar{V}) \mathrm{d} \zeta \mathrm{d} \Omega \mathrm{d} t \\
\quad=\int_{t_{i}}^{t_{f}} \int_{\Omega} \int_{h}\left[\rho\left(\delta \underline{u}+\zeta \delta \underline{E}_{3}\right) \cdot\left(\underline{u}+\zeta \underline{\underline{E}}_{3}\right)+\delta E \cdot(E+\zeta H) \tau+\delta H \cdot \zeta E \tau\right] \mathrm{d} \zeta \mathrm{d} \Omega \mathrm{d} t
\end{aligned}
$$

Integrating through the thickness of the shell, we get

$$
\int_{t_{i}}^{t_{f}} \int_{\Omega}\left\{\delta \underline{u} \cdot\left[\underline{\dot{h}}-\left(\underline{N}_{1,1}+\underline{N}_{2,2}\right)\right]+\delta \underline{E} \cdot\left[\underline{g}_{f}-\left(\underline{M}_{1,1}+\underline{M}_{2,2}\right)+\underline{N}_{3}\right]\right\} \mathrm{d} \Omega \mathrm{d} t=0 .
$$

In this expression, $\underline{h}=m \underline{\dot{u}}+s^{*} \underline{E}_{3}$, and $\underline{g}=s^{*} \underline{\dot{u}}+I^{*} \underline{\underline{E}}_{3}$ are the linear and angular momentum vectors of the shell, respectively; the mass coefficients are defined as $m=\int_{h} \rho \mathrm{d} \zeta, s^{*}=\int_{h} \rho \zeta \mathrm{d} \zeta, I^{*}=\int_{h} \rho \zeta^{2} \mathrm{~d} \zeta$. The spatial in-plane forces are $\underline{N}_{\alpha}=\left(E \underline{N}_{\alpha x}^{*}+H \underline{M}_{\alpha}^{*}\right) / \sqrt{a_{r r o}}$, the out-of-plane forces $\underline{N}_{3}=E \underline{N}_{3}^{*}$, and the bending moments $\underline{M}_{\alpha}=\left(E \underline{M}_{\alpha}^{*}\right) / \sqrt{a_{\alpha \alpha}}$. The convected forces are $N^{*}=\left[\underline{N}_{1}^{*}, \underline{N}_{2}^{*}, \underline{N}_{3}^{*}\right]=\int_{h} \tau \mathrm{d} \zeta$, and the bending moments $M^{*}=\left[\underline{M}_{1}^{*}, \underline{M}_{2}^{*}, \underline{M}_{3}^{*}\right]=\int_{h} \tau \zeta \mathrm{d} \zeta$.

3. Energy Preserving Scheme. Discrete equations of motion that imply conservation laws for the total mechanical energy, linear momentum and angular momentum of the system will now be developed. Times $t_{i}$ and $t_{f}$ denote the initial and final times for a time step, respectively, and the subscripts $(\bullet)_{i}$ and $(\bullet)_{f}$ indicate quantities at $t_{i}$ and $t_{f}$, respectively. Furthermore, the subscript $(\bullet)_{m}$ is used to denote mid-point average quantities defined as

$$
(\bullet)_{m}=\frac{1}{2}\left[(\bullet)_{f}+(\bullet)_{i}\right]
$$

The following matrix identity will be used extensively

$$
A_{f}^{T} B_{f}-A_{i}^{T} B_{i}=\left(A_{f}-A_{i}\right)^{T} B_{m}+A_{m}^{T}\left(B_{f}-B_{i}\right) .
$$

Hamilton's principle, eq. (2.15), is now approximated in time in the the following manner

$$
\begin{aligned}
\int_{\Omega} \int_{h}\left\{\rho\left[\left(\underline{u}_{f}-\underline{u}_{i}\right)+\zeta\left(\underline{E}_{3 f}-\underline{E}_{3 i}\right)\right]\right. & \cdot\left[\frac{\underline{\underline{u}}_{f}-\underline{\underline{u}}_{i}}{\Delta t}+\zeta \frac{\dot{E}_{3 f}-\dot{E}_{3 i}}{\Delta t}\right] \\
& \left.+\left(E_{f}-E_{i}\right) \cdot\left(E_{m}+\zeta H_{m}\right) \tau_{a}+\left(H_{f}-H_{i}\right) \cdot \zeta E_{m} \tau_{a}\right\} \mathrm{d} \zeta \mathrm{d} \Omega=0 .
\end{aligned}
$$

The change in strain components from $t_{i}$ to $t_{f}$ is evaluated with the help of identity (3.2) to find

$$
\begin{aligned}
e_{f}-e_{i}= & \frac{1}{2}\left[\left(E_{f}-E_{i}\right)^{T}\left(E_{m}+\zeta H_{m}\right)+\left(E_{m}+\zeta H_{m}\right)^{T}\left(E_{f}-E_{i}\right)\right. \\
& \left.+\left(H_{f}-H_{i}\right)^{T} \zeta E_{m}+\zeta E_{m}^{T}\left(H_{f}-H_{i}\right)\right] .
\end{aligned}
$$

Over one time step, the strain components can be approximated as $e(\eta)=e_{m}+\eta\left(e_{f}-e_{i}\right) / 2$, where $\eta=$ $2\left(t-t_{m}\right) / \Delta t$ is the non-dimensional time. If the strain energy density function $\bar{V}$ is viewed as a function of the scalar variable $\eta$, the mean value theorem then implies the existence of a $\bar{\eta} \in[-1,1]$ such that

$$
\bar{V}_{f}=\bar{V}_{i}+\left.\frac{\partial \bar{V}}{\partial \rho}\right|_{\bar{\eta}} \frac{d e}{d \eta} 2=\bar{V}_{i}+\tau_{a} \cdot\left(e_{f}-e_{i}\right)
$$


This relationship defines the average second Piola-Kirchhoff stress tensor, $\tau_{a}=\partial \bar{V} / \partial e_{\bar{\eta}}$. Combining this result with eq. (3.4) then leads to

$$
\left(E_{f}-E_{i}\right) \cdot\left(E_{m}+\zeta H_{m}\right) \tau_{a}+\left(H_{f}-H_{i}\right) \cdot \zeta E_{m} \tau_{n}=\left(e_{f}-e_{i}\right) \cdot \tau_{n}=\bar{\tau}_{f}-\bar{\tau}_{i}
$$

where the symmetry of the stress tensor was taken into account. For linear constitutive laws of the form $\tau=C^{*} e$, where $C^{*}$ is the stiffness matrix, the average stress tensor simply becomes $\tau_{n}=C^{*} e_{m}$.

The following configuration updates are now defined

$$
\frac{\underline{u}_{f}-\underline{u}_{i}}{\Delta t}=\underline{\underline{u}}_{m} ; \quad \frac{\underline{E}_{3 f}-\underline{E}_{3 i}}{\Delta t}=\underline{\dot{E}}_{3 m}
$$

Introducing eqs. (3.6) and (3.7) into the approximate expression for Hamilton's principle, eq. (3.3), then leads to

$$
\begin{aligned}
\int_{\Omega 2} \int_{h}\left\{\frac{\rho}{2}\left(\underline{\dot{u}}_{m}+\zeta \underline{\dot{E}}_{3 m}\right) \cdot\left[\left(\underline{\dot{u}}_{f}-\underline{\dot{u}}_{i}\right)+\zeta\left(\underline{\dot{E}}_{3 f}-\underline{\dot{E}}_{3 i}\right)\right]+\left(\bar{V}_{f}-\bar{V}_{i}\right)\right\} \mathrm{d} \zeta \mathrm{d} \Omega \\
=\int_{\Omega} \int_{h}\left\{\frac{\rho}{2}\left(\underline{\dot{u}}_{f}+\zeta \underline{\dot{E}}_{3 f}\right) \cdot\left(\underline{\dot{u}}_{f}+\zeta \underline{\dot{E}}_{3 f}\right)-\frac{\rho}{2}\left(\underline{\dot{u}}_{i}+\zeta \underline{\dot{E}}_{3 i}\right) \cdot\left(\underline{\underline{u}}_{i}+\zeta \underline{\dot{E}}_{3 i}\right)+\left(\bar{V}_{f}-\bar{V}_{i}\right)\right\} \mathrm{d} \zeta \mathrm{d} \Omega \\
\quad=\int_{\Omega} \int_{h}\left[\left(\bar{K}_{f}-\bar{K}_{i}\right)+\left(\bar{V}_{f}-\bar{V}_{i}\right)\right] \mathrm{d} \zeta \mathrm{d} \Omega=0 .
\end{aligned}
$$

This result clearly implies the conservation of the total mechanical energy of the system within a step. In summary, the approximate form of Hamilton's principle given by eq. (3.3) leads to a discrete energy conservation statement, eq. (3.8), when the configuration updates are chosen according to eqs. (3.7), and the average stress according to eq. (3.5).

Integrating through the thickness of the shell leads to

$$
\begin{aligned}
\int_{\Omega 2}\left\{( \underline { u } _ { f } - \underline { u } _ { i } ) \cdot \left[\frac{\underline{h}_{f}-\underline{h}_{i}}{\Delta t}-\left(\underline{N}_{1 m, 1}\right.\right.\right. & \left.\left.+\underline{N}_{2 m, 2}\right)\right] \\
& \left.+\left(\underline{E}_{3 f}-\underline{E}_{3 i}\right) \cdot\left[\frac{\underline{g} f-\underline{g}_{i}}{\Delta t}-\left(\underline{M}_{1 m, 1}+\underline{M}_{2 m, 2}\right)+\underline{N}_{3 m}\right]\right\} \mathrm{d} \Omega=0 .
\end{aligned}
$$

In this expression, the in-plane forces are $\underline{N}_{\alpha m}=\left(E_{m} \underline{N}_{\alpha a}^{*}+H_{m} \underline{M}_{\alpha a}^{*}\right) / \sqrt{a_{\alpha \alpha}}$, the out-of-plane forces $\underline{N}_{3 m}=$ $E_{m} \underline{N}_{3 a}^{*}$, and the bending moments $M_{\alpha m}=\left(E_{m} \underline{M}_{\alpha a}^{*}\right) / \sqrt{\Omega_{\alpha \alpha}}$. The discrete governing equations of motion for shells are then

$$
\begin{gathered}
\frac{\underline{h}_{f}-\underline{h}_{i}}{\Delta t}-\left(\underline{N}_{1 m, 1}+\underline{N}_{2 m, 2}\right)=\underline{p}_{m} ; \\
Q_{m}^{T} \frac{\underline{g} f-\underline{g}_{i}}{\Delta t}-Q_{m}^{T}\left(\underline{M}_{1 m, 1}+\underline{M}_{2 m, 2}-\underline{N}_{3 m}\right)=\underline{q}_{m}^{*},
\end{gathered}
$$

where $\underline{p}$ are the externally applied loads, and $\underline{q}^{*}$ the externally applied moments measured in the local system. The finite change in director orientation $\underline{E}_{3 f}-\underline{E}_{3 i}$ was expressed in terms of the two parameter incremental rotation vector, see $B$.

Invariance of the system Hamiltonian under spatial translations and rotations implies the conservation of the linear and angular momenta. The preservation of these invariants is less crucial from a numerical point of view, since it does not lead to non-linear notions of stability as in the case of the energy. However, is can be of some interest to note that momenta are indeed preserved at the discrete solution level by eqs. (3.10), (3.11) and (3.7). The complete proof of this assertion is reported in ref. [12], and it is based on projecting the discrete equations of dynamic equilibrium onto suitable test functions and integrating over the shell. 
Finally, it is important to point out that the particular spatial discretization adopted plays no role in the proofs leading to the properties of discrete energy and momentium conservation. Therefore, any spatial discretization of the discrete equations of motion will inherit these properties, when the configuration updates are chosen according to eqs. (3.7), and the average stress according to eq. (3.5).

4. Energy Decaying Scheme. In this section we derive a scheme associated to a discrete law of energy decay, using the EP scheme as a basic building block. The resulting integrator for shells features high frequency numerical damping, overcoming the difficulties and lack of robustness of EP methods.

First, an additional state is introduced at time $t_{j}=\lim _{\mathfrak{c} \rightarrow 0}\left(t_{i}+\epsilon\right)$, and the subscript $(\bullet)_{j}$ is used to denote quantities at this time. The following averages are now defined

$$
(\bullet)_{g}=\frac{1}{2}\left[(\bullet)_{f}+(\bullet)_{j}\right] ; \quad(\bullet)_{h}=\frac{1}{2}\left[(\bullet)_{j}+(\bullet)_{i}\right] .
$$

The ED scheme proceeds from the initial to the final time by means of two coupled steps: one step from $t_{i}$ to $t_{f}$, the other from $t_{i}$ to $t_{j}$. The time-discrete equations of dynamic equilibrium are

$$
\begin{gathered}
\frac{\underline{h}_{f}-\underline{h}_{i}}{\Delta t}-\left(\underline{N}_{1 g, 1}+\underline{N}_{2 g, 2}\right)=\underline{p}_{g} ; \\
Q_{m}^{T} \frac{\underline{g}_{f}-\underline{g}_{i}}{\Delta t}-Q_{g}^{T}\left(\underline{M}_{1 g, 1}+\underline{M}_{2 g, 2}-\underline{N}_{3 g}\right)=\underline{q}_{g}^{*} ; \\
\frac{\underline{h}_{j}-\underline{h}_{i}}{\Delta t}+\frac{1}{3}\left[\left(\underline{N}_{1 g, 1}+\underline{N}_{2 g, 2}\right)-\left(\underline{N}_{1 p, 1}+\underline{N}_{2 p, 2}\right)\right]=\underline{p}_{h} ; \\
Q_{h}^{T} \frac{\underline{g}_{j}-\underline{g}_{i}}{\Delta t}+\frac{1}{3}\left[Q_{g}^{T}\left(\underline{M}_{1 g, 1}+\underline{M}_{2 g, 2}-\underline{N}_{3 g}\right)-Q_{h}^{T}\left(\underline{M}_{1 p, 1}+\underline{M}_{2 p, 2}-\underline{N}_{3 p}\right)\right]=\underline{q}_{h}^{*} .
\end{gathered}
$$

The configuration update relationships are given as

$$
\begin{array}{ll}
\underline{u}_{f}=\underline{u}_{i}+\Delta t\left(\underline{\dot{u}}_{f}+\underline{\dot{u}}_{j}\right) / 2, & \underline{u}_{j}=\underline{u}_{i}-\Delta t\left[\underline{\dot{u}}_{f}-\underline{\dot{u}}_{i}-\alpha\left(\underline{\dot{u}}_{j}-\underline{\dot{u}}_{i}\right)\right] / 6 ; \\
\underline{E}_{3 f}=\underline{E}_{3 i}+\Delta t\left(\underline{\dot{E}}_{3 f}+\underline{\dot{E}}_{3 j}\right) / 2, & \underline{E}_{3 j}=\underline{E}_{3 i}-\Delta t\left[\underline{\dot{E}}_{3 f}-\underline{\dot{E}}_{3 i}-\alpha\left(\underline{\dot{E}}_{3 j}-\underline{\dot{E}}_{3 i}\right)\right] / 6,
\end{array}
$$

where $\alpha$ is a tuning parameter that controls the amount of numerical dissipation provided by the scheme, while the forces $\underline{N}_{\alpha p}$ and moments $\underline{M}_{\alpha p}$ are given by

$$
\underline{N}_{\alpha p}=\underline{N}_{\alpha h}+\alpha\left(\underline{N}_{\alpha j}-\underline{N}_{\alpha i}\right) / 2 ; \quad \underline{M}_{\alpha p}=\underline{M}_{\alpha h}+\alpha\left(\underline{M}_{\alpha j}-\underline{M}_{\alpha i}\right) / 2 .
$$

Using developments similar to those exposed for the EP scheme, it can be easily shown that the proposed discrete equations imply

$$
\left(K_{f}+V_{f}\right)-\left(K_{i}+V_{i}\right)+\alpha c^{2}=0
$$

$c^{2}$ is a positive quantity given by

$$
\begin{aligned}
c^{2} & =\int_{\Omega} \frac{1}{2}\left[m\|\underline{\dot{u}}\| \cdot\|\underline{\dot{u}}\|+2 s^{*}\|\underline{\dot{u}}\| \cdot\left\|\underline{\dot{E}}_{3}\right\|+I^{*}\left\|\underline{\dot{E}}_{3}\right\| \cdot\left\|\underline{\dot{E}}_{3}\right\|\right] \mathrm{d} \Omega \\
& +\int_{\Omega} \frac{1}{2}\|\underline{e}\| C^{*}\|\underline{e}\| \mathrm{d} \Omega \geq 0
\end{aligned}
$$

where $\|\bullet\|=(\bullet)_{j}-(\bullet)_{l}$ is the jump between $t_{i}$ and $t_{j}$. This result implies the decay of the total mechanical energy over one step of the algorithm, $\left(K_{f}+V_{f}\right) \leq\left(K_{i}+V_{i}\right)$. The parameter $\alpha$ can be used for controlling 


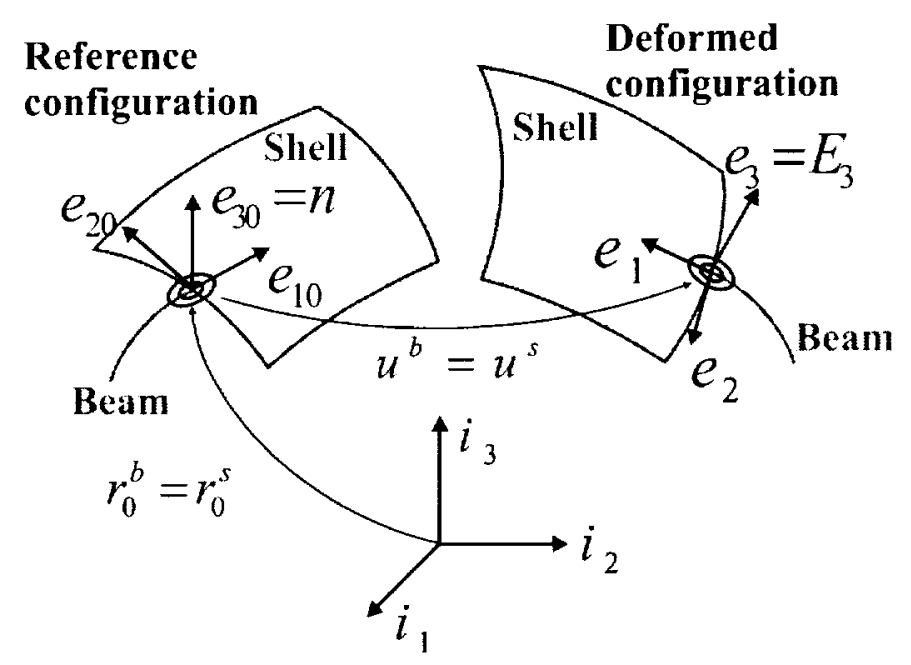

FIG. 5.1. Configuration of the shell revolute joint in the reference and deformed configurations.

the amount of energy that is dissipated within the step. It should be noted that the property of preservation of momentum observed in the EP case is lost in the FD algorithm.

It is interesting to characterize the ED scheme with the classical tools of linear analysis. In fact, if the scheme is applied to a single degree of freedom linear oscillator model problem, the asymptotic value of the spectral radius of the amplification matrix, $\rho_{\infty}$, is found to be $\rho_{\infty}=(1-\alpha) /(1+\alpha)$. For $\alpha=1, \rho_{\infty}=0$, and asymptotic annihilation is achieved. If $\alpha=0, \rho_{\infty}=1$, and in view of eq. (4.6), energy is exactly preserved. Hence, the ED scheme is in fact a family of schemes with a single tuning parameter, $\alpha$, that controls the amount of high frequency numerical dissipation; both asymptotic annihilation or exact energy preservation can be achieved with the same scheme by using $\alpha=1$ or 0 , respectively.

5. The Shell Revolute Joint. When modeling multibody systems with shell components, connections between shell elements and other elements of the model must be carefully treated. Indeed, beam or joint nodes involve six degrees of freedom, three displacements and three rotations. On the other hand, shell nodes involve five degrees of freedom, three displacements and two rotations that determine the orientation of the director $\underline{E}_{3}$. A typical situation is shown in fig. 5.1 that depicts the connection between a beam and a shell. If the beam were clamped to the shell, the beam would apply bending moments and a "drilling moment" at the connection point. The shell cannot sustain such drilling moment since it presents no stiffness about an axis normal to its reference surface. Hence, the connection between the beam and the shell must be done through a shell revolute joint, i.e. a revolute joint with its axis of rotation perpendicular to the shell reference surface.

Consider a beam and a shell denoted with superscripts $(.)^{k}$ and $(.)^{l}$, respectively, linked together by a shell revolute joint, as depicted in fig. 5.1. In the reference configuration, the normal to the shell is $\underline{n}$ and the triad $\underline{e}_{10}, \underline{e}_{20}$, and $\underline{e}_{30}=\underline{n}$ is attached to the beam at the connection point. In the deformed configuration, no relative displacements are allowed and the beam attached triad rotates to $\underline{e}_{1}, \underline{e}_{2}$, and $\underline{e}_{3}=\underline{E}_{3}$. This condition implies the orthogonality of $\underline{E}_{3}$ to both $\underline{e}_{1}$ and $\underline{e}_{2}$. The kinematic constraints associated with a shell revolute joint are

$$
\mathcal{C}_{\curvearrowright}=\underline{E}_{3} \cdot \underline{e}_{\alpha}=0
$$

where $\alpha=1,2$. Of course, at the connection point, the displacements of the beam and shell are identical; this 


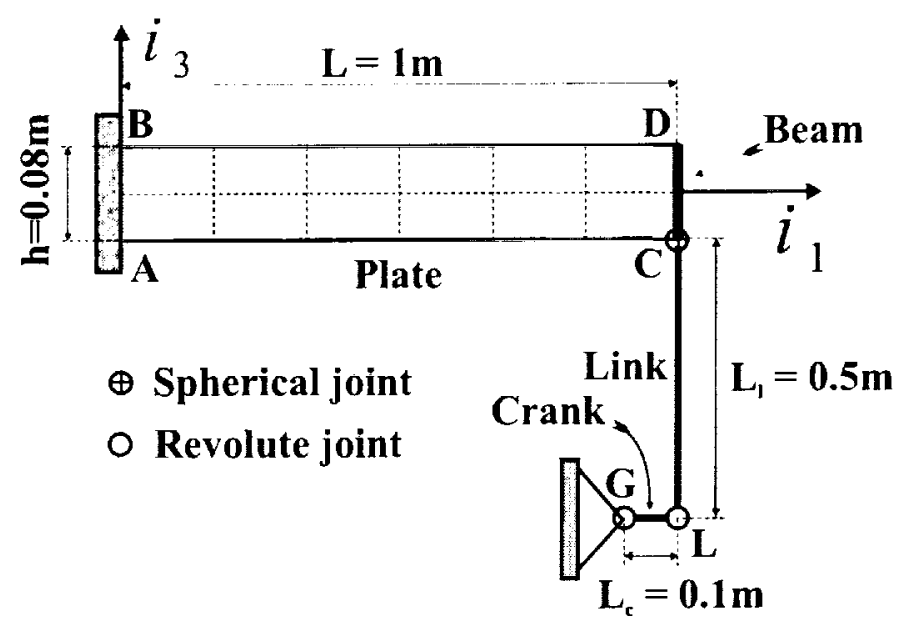

FIG. 6.1. Configuration of the lateral buckling problem.

constraint is readily enforced within the framework of finite element formulations by Boolean identification of the corresponding degrees of freedom. Holonomic constraints are enforced by the addition of a constraint potential $\lambda \mathcal{C}$, where $\lambda$ is the Lagrange multiplier. The details of the procedure used to enforce constraints in such a manner that the work done by the constraint forces vanishes exactly can be found in refs. [3,5].

6. Numerical Examples. All the examples described in this section will be treated with the proposed ED family of schemes corresponding to values of the tuning parameter $\alpha \in[0,1]$. Although any value of $\alpha$ within this range can be used, the examples clescribed here will contrast the two extreme choices. For $\alpha=1$ $\left(\rho_{\infty}=0\right)$, asymptotic annihilation is obtained, and this will be called the ED scheme. On the other hand, for $\alpha=0\left(\rho_{\infty}=1\right)$, exact energy preservation is achieved, and this will be called the FP scheme.

6.1. Lateral Buckling of a Thin Plate. Consider a thin cantilevered plate acted upon by a crank and link mechanism, as depicted in fig. 6.1. The plate is of length $L=1 \mathrm{~m}$, width $h=0.08 \mathrm{~m}$ and thickness $t=2 \mathrm{~mm}$. It is clamped along edge $A B$ and reinforced along edge $C D$ by a beam with a square cross-section of side $a=4 \mathrm{~mm}$. At point $C$, the beam connects to a crank and link mechanism. The crank of length $L_{c}=0.1 \mathrm{~m}$ is attached to the ground at point $G$, and the link is of length $L_{c}=0.5 \mathrm{~m}$. The ground, crank, and link are connected together by means of revolute joints, whereas the beam and link are connected through a spherical joint. All components are made of steel with the following properties: Young's modulus $E=210 \mathrm{GPa}$, Poisson ratio $\nu=0.25$ and density $\rho=7870 \mathrm{~kg} / \mathrm{m}^{3}$. The crank rotates at constant angular velocity $\Omega=1 \mathrm{rad} / \mathrm{s}$, and the system is simulated for a period of $2 \pi \mathrm{s}$, corresponding to a complete revolution of the crank.

The system is modeled first using a geometrically exact beam element, then using the shell element described in this paper. The crank and link are modeled by rigid bodies. For the beam model, three fournoded, geometrically exact beam elements were used, whereas for the shell model a $6 \times 2$ grid of quadratic elements was used. The simulation used the ED version of the proposed algorithm $\left(\rho_{\infty}=0.0\right)$ with a constant time step $\Delta t=10^{-03} \mathrm{~s}$.

As the crank rotates, the plate deflects downwards then snaps laterally when its buckling load is reached. In the post-buckling regime, the plate becomes significantly softer in bending due to its large twisting allowed by the spherical joint. These features are illustrated in fig. 6.2 that depicts the post-buckling of the plate at various times. The plate mid-span lateral deflections obtained for both beam and shell models are shown in 


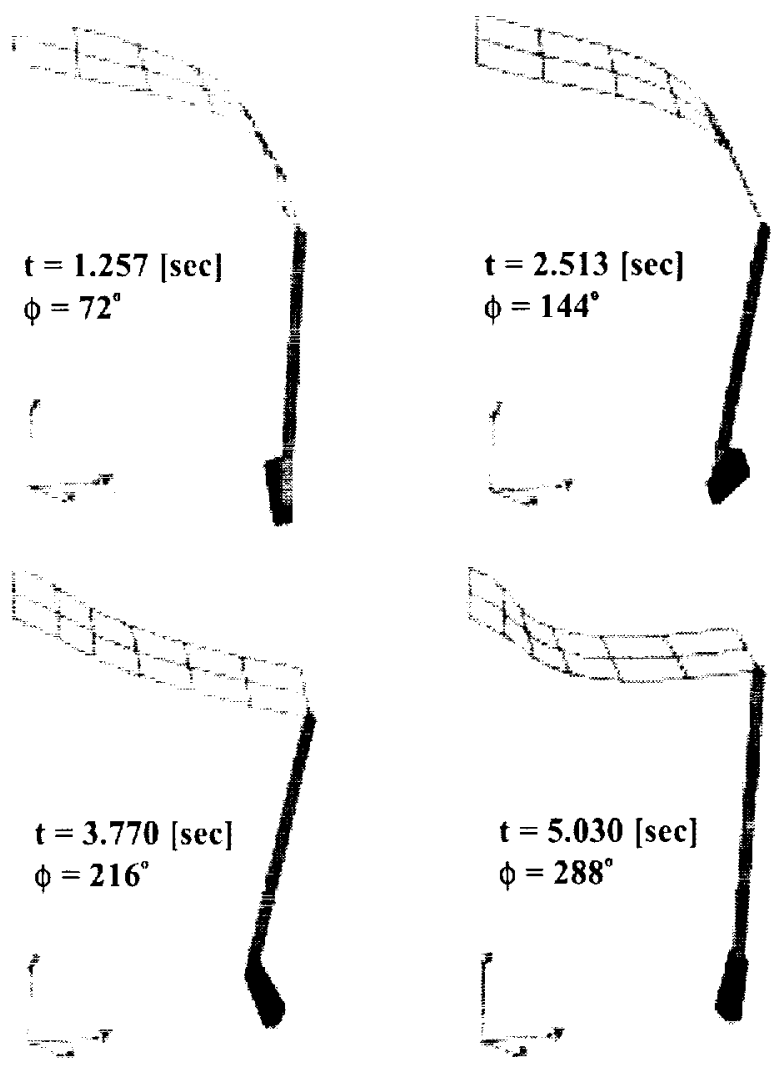

FiG. 6.2. System configurations at various time instants during the simulation.

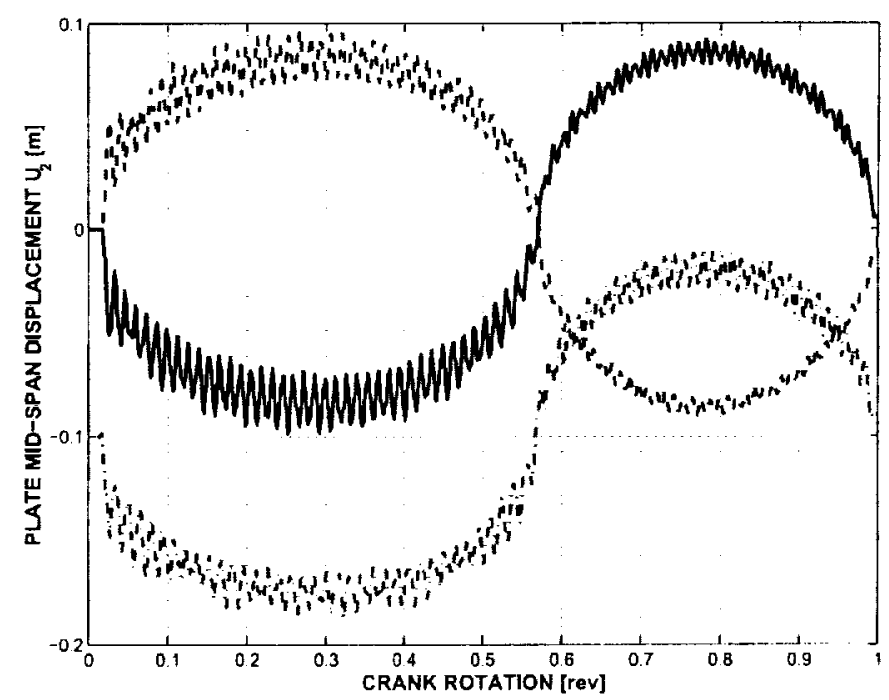

FIG. 6.3. Time history of the mid-span lateral displacement of the plate. Shell model (ED scheme): solid line; shell model (FP scheme): dashed line; beam model: dash-dotted line (results shifted downwards $0.1 \mathrm{~m}$ for clarity). 


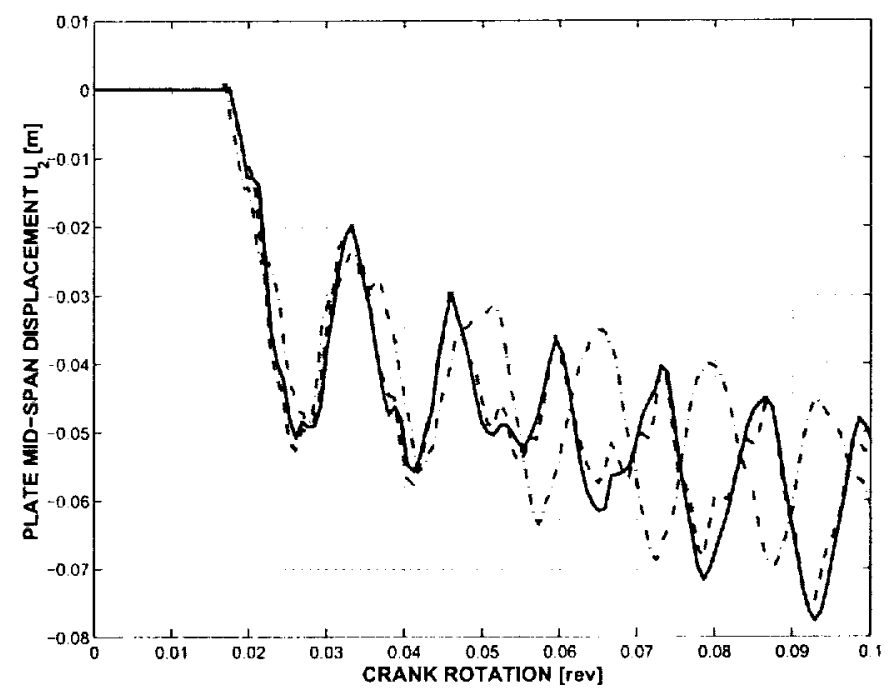

FIG. 6.4. Time history of the mid-span lateral displacement of the plate. Shell model (ED scheme): solid line; shell model (EP scheme): dashed line (the sign of the displacement was changed to ease the comparison); beam model: dash-dotted line.

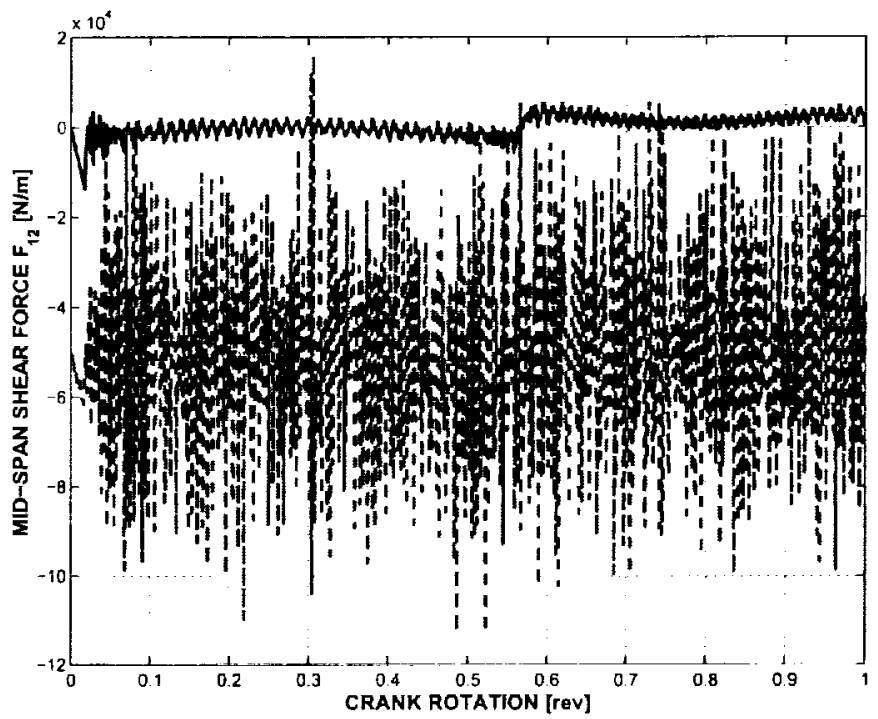

FIG. 6.5. Time history of the shell mid-span in-plane shear force $F_{12}$. ED scheme: solid line; EP scheme: dashed line (results shifted downwards $510^{4} \mathrm{~N} / \mathrm{m}$ for clarity).

fig. 6.3, and good agreement between the models is observed. In both cases, elastic vibrations are superposed onto the overall motion imparted by the crank. An enlarged view of the onset of buckling for both models is shown in fig. 6.4; the sudden appearance of lateral deflections and subsequent oscillations are observed. This figure also shows that the agreement between the beam and shell models is qualitative, not quantitative, as should be expected.

Prior to buckling, the plate resists the bending loads applied by the driving mechanism with very little deformations, and high shear forces build up in the plate. When buckling occurs, twisting of the plate renders it much softer in the vertical direction, offering little resistance to crank motion. Figs. 6.5 and 6.6 show the mid-span in-plane shear force $F_{12}$ and crank driving torque, respectively. Note the linear increase 

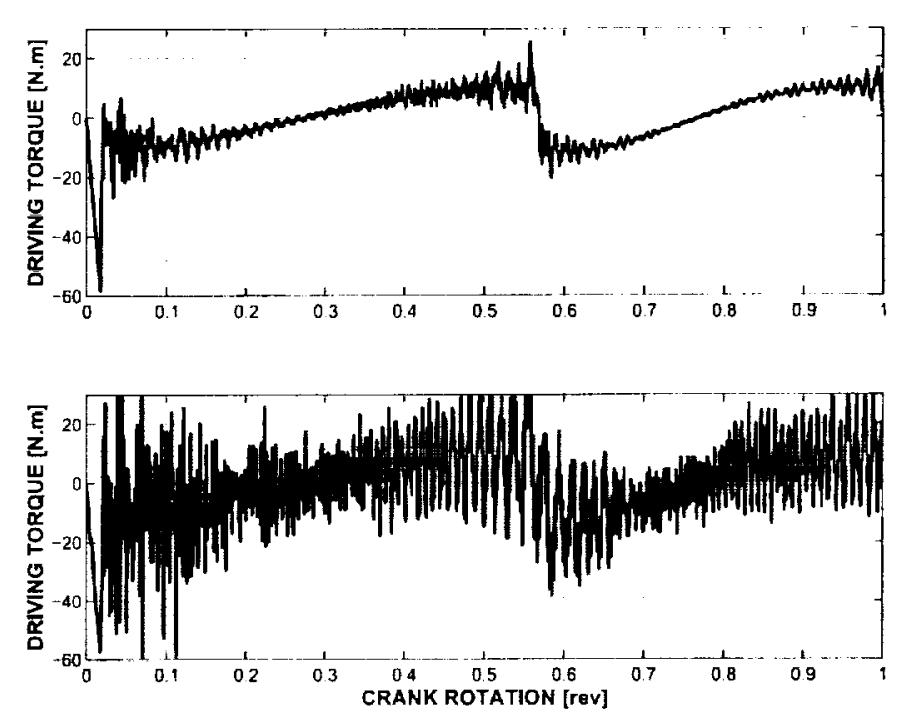

FIG. 6.6. Time history of the crank driving torque. FD scheme: top figure; FP scheme: bottom figure.

of the driving torque up to $Q \approx 60 \mathrm{Nm}$, followed by an abrupt drop at buckling. This jump excites the vibratory modes of the system.

Next, the same problem was simulated using the EP schemes, i.e. $\left(\rho_{\infty}=1.0\right)$. Lateral displacements, see figs. 6.3 and 6.4, are found to be in good agrement with the ED predictions. The change in sign for the lateral deflection is immaterial since the plate can buckle in either direction. Figs. 6.5 and 6.6 compare the predictions of the EP and ED schemes for mid-span in-plane shear force $F_{12}$ and crank driving torque, respectively. The EP predictions show high frequency oscillations with amplitudes an order of magnitude larger than those predicted by the ED scheme. This numerical noise completely obscures the results of the computation.

6.2. Snap-Through of a Cylindrical Shell. A crank and link mechanism is used to drive a cylindrical shell through an unstable, snap-through configuration. The system geometry is depicted in fig. 6.7. The shell consists of a 60 degree sector of a cylinder of height $h=2.5 \mathrm{~m}$, radius $R=5 \mathrm{~m}$ and thickness $t=8 \mathrm{~mm}$. The two straight edges of the shell are simply supported, whereas the other two are free. The shell is reinforced along line $B E$ by a beam with a square cross-section of side $a=20 \mathrm{~mm}$. At point $E$, the beam connects to a crank and link mechanism. The crank of length $L_{c}=1.5 \mathrm{~m}$ is attached to the ground at point $G$ located $5 \mathrm{~m}$ below point $E$. The beam, link, crank, and ground are connected together by means of revolute joints. The crank is modeled as a rigid body, while the link is a beam with a square cross-section of side $s=40 \mathrm{~mm}$. The shell and reinforcing beam are made of aluminum: Young's modulus $E=73 \mathrm{GPa}$, Poisson ratio $\nu=0.30$ and density $\rho=2700 \mathrm{~kg} / \mathrm{m}^{3}$. The lever is made of steel: Young's modulus $E=210 \mathrm{GPa}$, Poisson ratio $\nu=0.30$ and density $\rho=7800 \mathrm{~kg} / \mathrm{m}^{3}$.

The crank rotates with the following schedule

$$
\Phi(t)=\left\{\begin{array}{ll}
\pi(1-\cos 2 \pi t / T) / 2 & t \leq T / 2 \\
\pi & t>T / 2
\end{array},\right.
$$

where $T=3 \mathrm{~s}$. The system is simulated for a period of $2 \mathrm{~s}$. The shell is meshed with an $8 \times 4$ grid of quadratic: shell elements, whereas the link is meshed with four cubic beam elements. The simulation was conducted using the ED scheme with $\rho_{\infty}=0$, i.e. asymptotic annihilation, and a constant time step size 


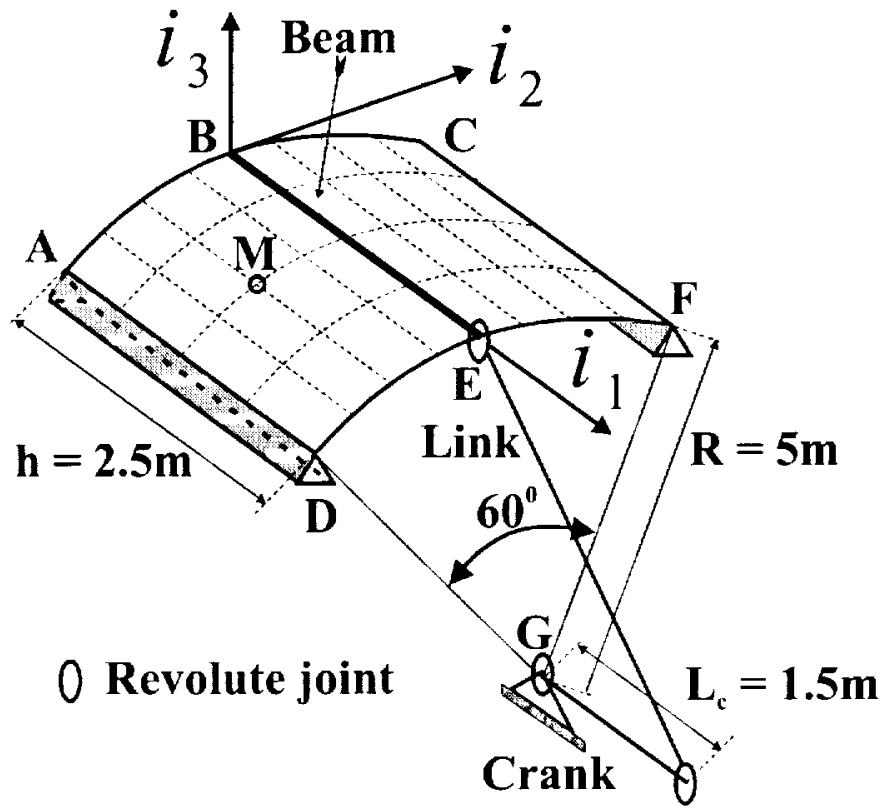

FiG. 6.7. Configuration of the snap-through problem.
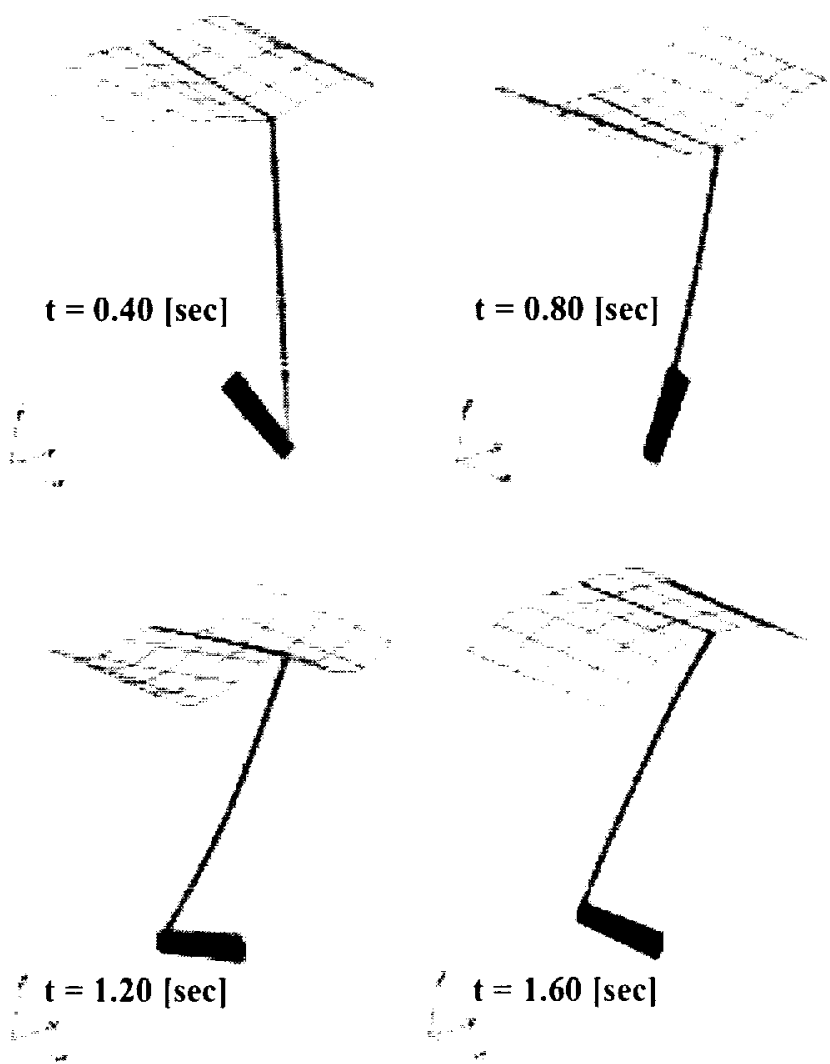

FiG. 6.8. System configurations at various instants in time. 


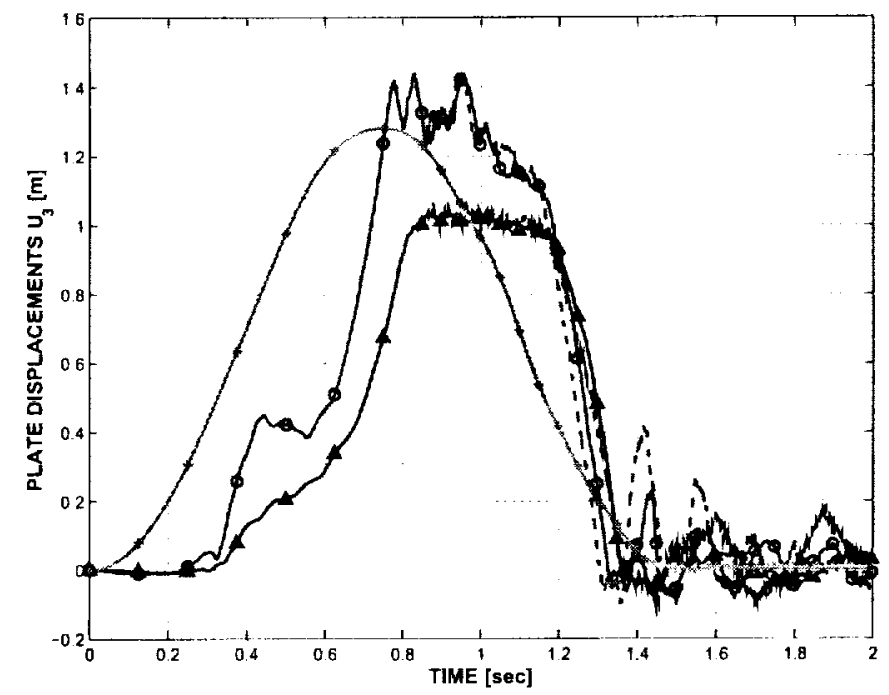

Fig. 6.9. Time history of the vertical displacements at points $B(0), M(\triangle)$ and $E(+)$. ED scheme: solid line; FP scheme: dashed line.

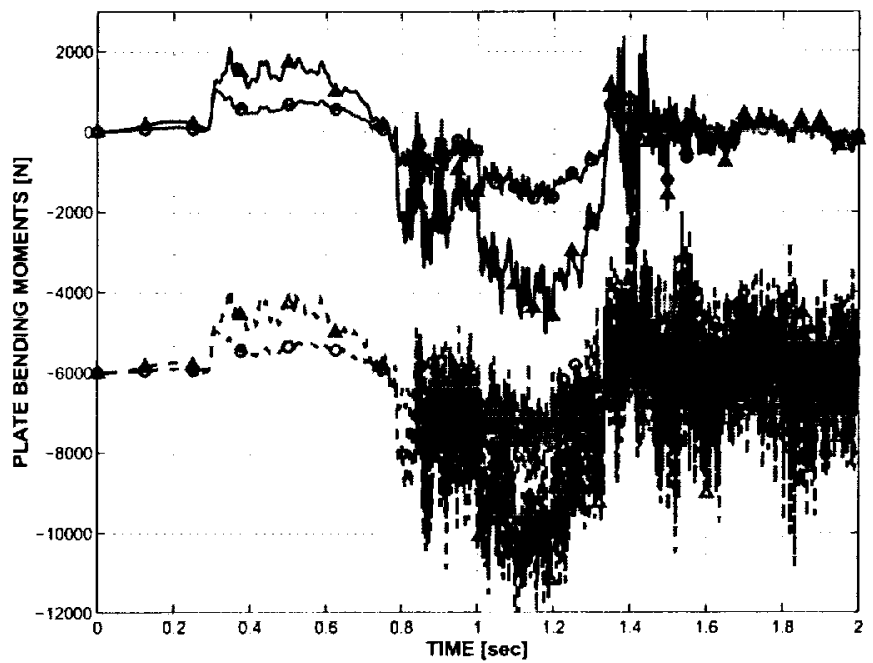

Fici. 6.10. Time history of the bending moments $M_{11}$ (O) and $M_{22}(\triangle)$ at point $F$. FD scheme: solid line; FP scheme: dashed line. For clarity, the FP predictions were shifted doun by $6 \mathrm{kN}$.

$\Delta t=2.510^{-3} \mathrm{~s}$.

During the first 90 degree rotation of the crank, the link pulls the shell downwards until snap-through takes place and curvature reverses. Curvature reversal initiates in the neighborhood of the link connection, then quickly propagates to the shell free edge, which undergoes violent oscillations. During the next 90 degree rotation of the crank, the link now pushes the inverted shell upwards, until snap-through occurs and curvature reverts to its original sign. During the entire sequence, violent oscillations are observed, as depicted in fig. 6.8 that shows the system at various instants in time.

Vertical displacements at point $B, M$, and $E$ are shown in fig. 6.9. The displacement of point $E$ closely follows the prescribed input imparted by the crank, whereas those at points $B$ and $M$ reflect the additional elastic vibrations of the shell. At first, the shell takes a double-S configuration: the curvature of the central part of the cylinder is already negative, while remaining positive along the simply supported edges. Snap- 

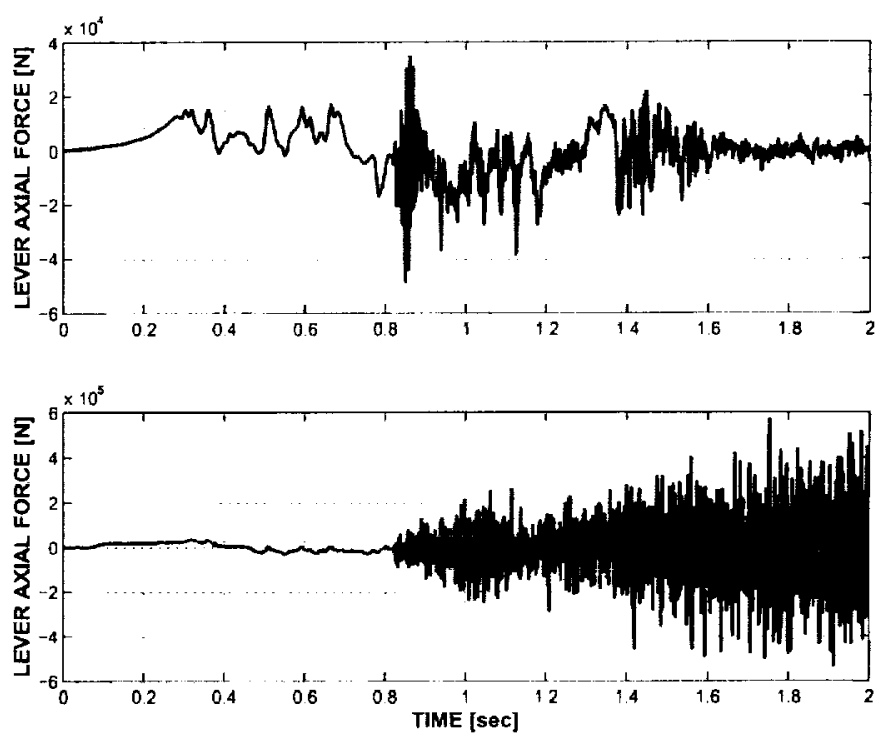

FIG. 6.11. Time history of the lever mid-span axial force. Fn scheme: top figure; EP scheme: bottom figure. Note the different, scales for the two figures.
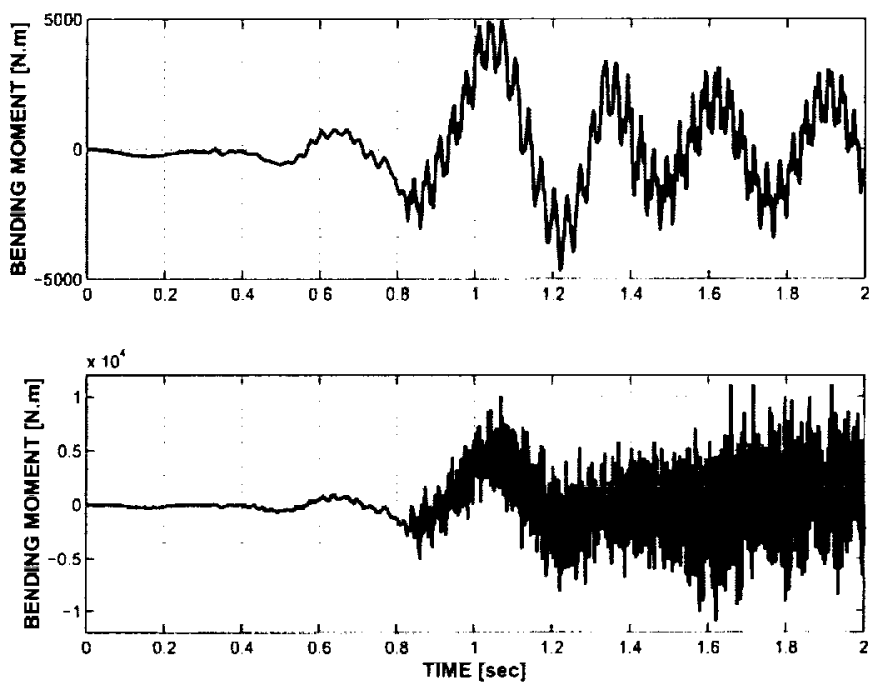

FlG. 6.12. Time history of the lever mid-span bending moment. FD scheme: top figure; EP scheme: bottom figure. Note the different scales for the two figures.

through occurs at about $t \approx 0.7 \mathrm{~s}$ and vibrations occur in the inverted configuration. As the crank pushes the shell back up, local deformations appear at first, followed by a rapid snap-back to the original curvature at $t \approx 1.25 \mathrm{~s}$, when the crank is about to stop. Due to the speed of snap-back, violent oscillations about the original shell configuration are observed in the latter part of the simulation. The components $M_{11}$ and $M_{22}$ of bending moment at point $E$ are shown in fig. 6.10. Here again, the snap-through events are clearly identifiable. The lever mid-span axial force and bending moment are sown in figs. 6.11 and 6.12, respectively,

Next, the system was simulated with the EP scheme, i.e. with $\rho_{\infty}=1$. In order to achieve convergence of the iterative solution procedure for the nonlinear equations of motion, the time step size had to be reduced to $\Delta t=1.2510^{-03}$ then $6.2510^{-04} \mathrm{~s}$ at times $t=0.835$ and $0.8475 \mathrm{~s}$, respectively. A good agreement 


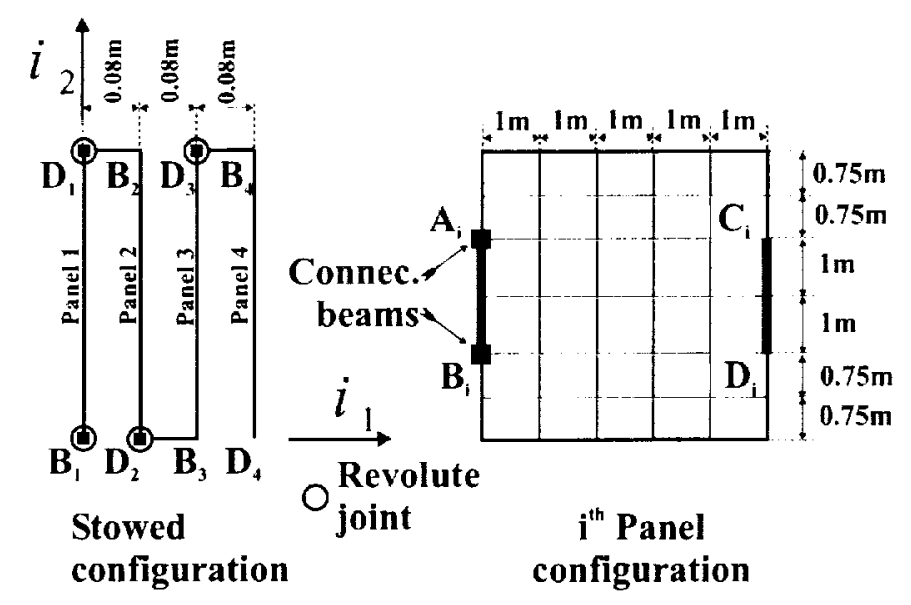

FIc: 6.13. Configuration of the space antenna deployment problem.

between the ED and EP predictions is shown in fig. 6.9, although discrepancies are apparent when significant oscillations occur. The bending moments shown in fig. 6.10 are once again in good agreement during the beginning of the simulation, but at the end of the snap-through phase (i.e. $t \approx 0.835 \mathrm{~s}$ ), the EP scheme is unable to deal with the complex dynamic behavior of the system: a smaller time step is required for convergence, and violent high frequency oscillations of a purely numerical origin appear. The amplitudes of these oscillations are an order of magnitude larger than those predicted by the ED scheme. The same remarks can be made about the lever mid-span axial force and bending moments shown in figs. 6.11 and 6.12, respectively. In fact, the bending moment response, a simple superposition of oscillations involving the lowest two natural modes of the lever as predicted by the ED scheme, is completely obscured by numerical noise in the EP scheme. Since vibratory stresses are of great importance to designers, it is essential to assess the ability of new integration schemes to reliably predict these quantities. It is unfortunate that many scientific publications about geometric integration only present responses for preserved quantities such as total mechanical energy or momentum. The above plots demonstrate that while EP schemes might perform very well for the prediction of total energy, momentum, or even displacement fields, they are unable to reliably predict other important fields such as velocities and internal stresses. Consequently, such schemes are of little value in real life applications.

6.3. Deployment of a Space Antenna. Consider a space antenna consisting of four square composite panels of size $5 \times 5 \mathrm{~m}$. A typical panel in shown in fig. 6.13. Each panel is reinforced along opposite edges $A_{i} B_{i}$ and $C_{i} D_{i}$ by beams of length $L=2 \mathrm{~m}$. These reinforcing beams are attached at points $A_{i}$ and $B_{i}$ to connector beams, and points $C_{i}$ and $D_{i}$ to revolute joints. The connector beams are attached to the revolute joints of the preceding panel. At points $A_{1}$ and $B_{1}$, Panel 1 is connected to the ground by means of revolute joints. The stowed configuration of the entire system is also depicted in fig. 6.13.

The panels are made of laminated composite material with a 12 ply lay-up $\left[0,90,45,-45,45,-45^{\circ}\right]_{s}$, where the $90^{\circ}$ direction is parallel to that of the rcinforcing beams. The material properties of the composite are: longitudinal modulus $E_{L}=138 \mathrm{GPa}$, transverse modulus $E_{T}=8.96 \mathrm{GPa}$, shearing modulus $G_{L T}=$ $7.1 \mathrm{GPa}$, Poisson's ratio $\nu_{I T}=0.3$, and ply thickness $t_{p}=0.125 \mathrm{~mm}$. The reinforcing and connector beams have a square cross-section of side $a_{r}=10 \mathrm{~mm}$ and $a_{c}=20 \mathrm{~mm}$, respectively. They are made of a homogeneous isotropic material with the following properties: $E=140 \mathrm{GPa}$, and density $\rho=7000 \mathrm{~kg} / \mathrm{m}^{3}$. Each of the eight revolute joints weighs $8 \mathrm{~kg}$ and is spring loaded to deploy the antenna. Each spring applies 


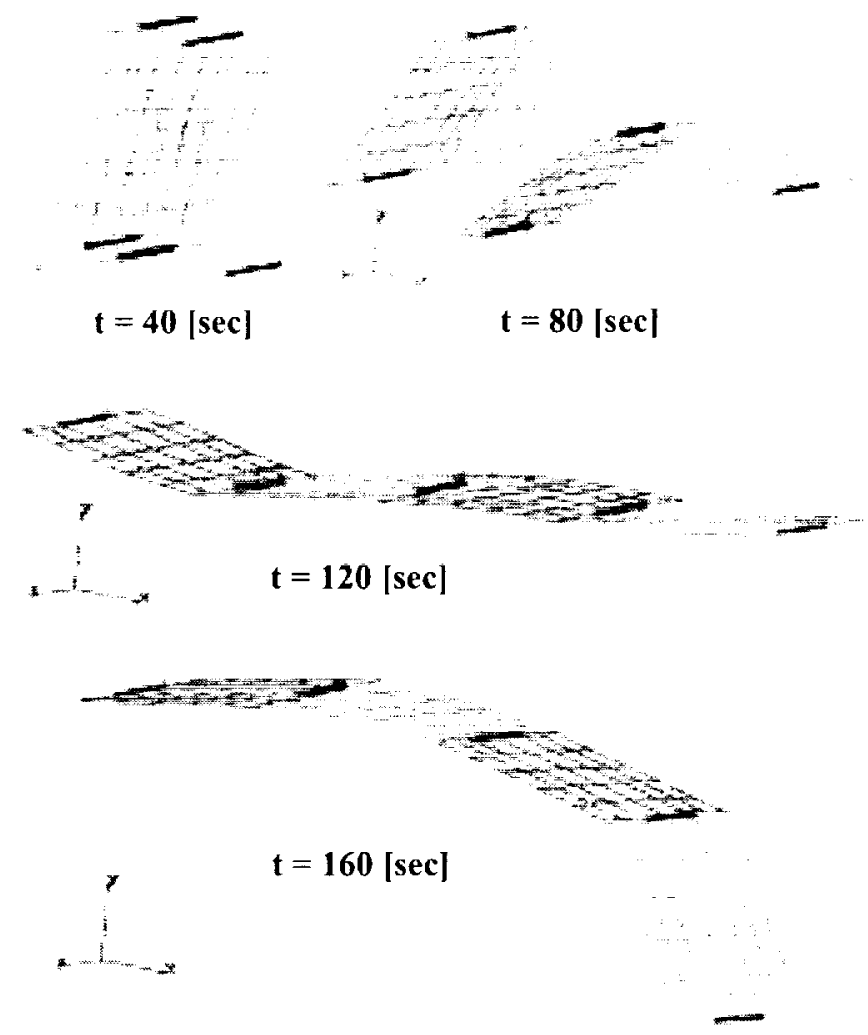

FIG. 6.14. Configuration of the space antenna at various instants in time.

TARLF, 6.1

Physical properties of the springs and dampers at the revolute joints.

\begin{tabular}{|c|c|c|c|c|}
\hline $\begin{array}{c}\text { Revolute } \\
\text { Joint at }\end{array}$ & $\begin{array}{c}M_{0} \\
{[\mathrm{Nm}]}\end{array}$ & $\begin{array}{c}\theta_{0} \\
{[\mathrm{rad}]}\end{array}$ & $n$ & $\begin{array}{c}\mu \\
\text { [Nms/rad] }\end{array}$ \\
\hline$A_{1}, B_{1}$ & 1.0 & $\pi / 2$ & 6 & 20.0 \\
$C_{1}, D_{1}$ & 0.8 & $\pi$ & 12 & 9.0 \\
$C_{2}, D_{2}$ & 0.7 & $\pi$ & 12 & 7.0 \\
$C_{3}, D_{3}$ & 0.5 & $\pi$ & 12 & 5.0 \\
\hline
\end{tabular}

a moment $M_{s}=M_{0}\left[1-\left(\theta / \theta_{0}\right)^{n}\right]$ at the joint. Furthermore, a damper is present in each joint and applies a moment $M_{d}=\mu \dot{\theta}$, where $\theta$ is the relative rotation at the joint. The constants $M_{0}, \theta_{0}, n$ and $\mu$ are listed in table 6.1 for each joint.

Under the effect of the springs in the revolute joint, the antenna deploys. Configurations of the system at various instants in time are shown in fig. 6.14, and the relative rotations at four revolute joints during deployment are shown in fig. 6.15. To validate the simulation, a simplified model of the system using geometrically exact beam elements to represent the pancls was also run. The predictions of both models are in fair agreement.

It should be noted that the composite lay-up used for the panels presents a $10 \%$ bending-twisting elastic: coupling term. Consequently, the bending of the panels during deployment generates twisting; and the motion of the entire system becomes three-dimensional. To illustrate this effect, the rotations of the 

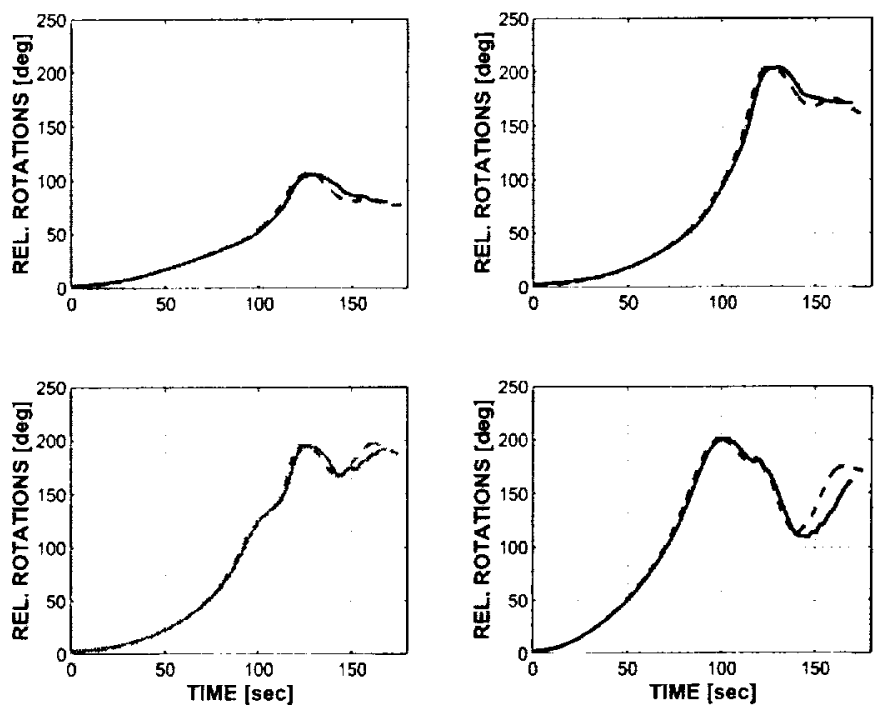

Fig. 6.15. Relative rotation at the revolute joints. Joint at point $B_{1}:$ top-left, $D_{1}$ : top-right, $D_{2}$ : bottom-left, and $D_{3}$ : bottom-right figure. Shell model: solid line; beam model: dashed line.
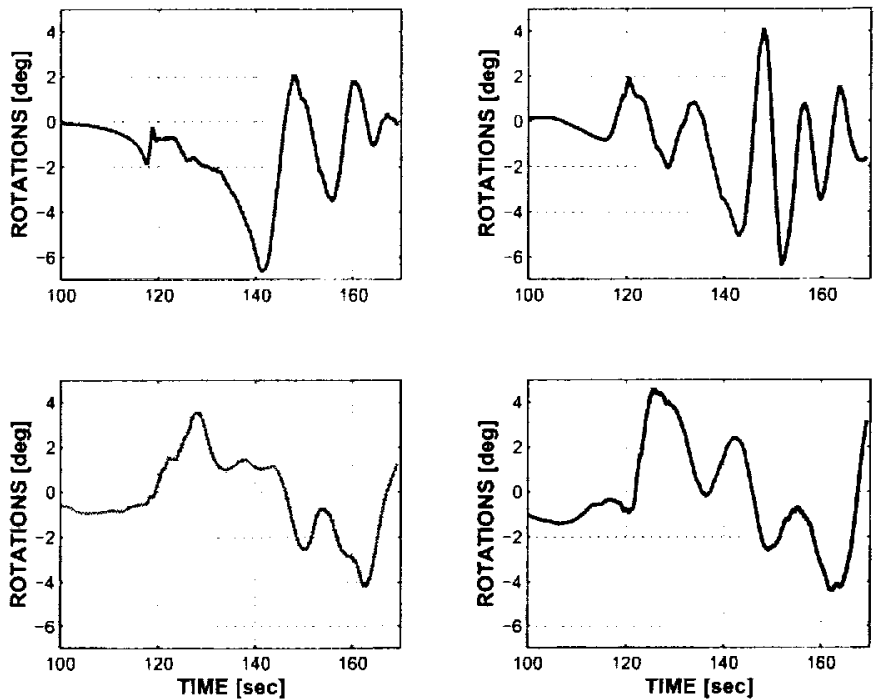

FIG 6.16. Rotation of the reinforcing beams about the $\mathbf{i}_{1}$ axis. Panel 1: top-left, Panel 2: top-right, Panel 3: bottom-left, Panel 4: bottom-right figure.

reinforcing beams about the axis $\mathbf{i}_{1}$ are shown in fig. 6.16 . These rocking motions of up to 7 degrees generate large, mid-span shear forces in the connecting beams between Panels 1 and 2 that are shown in fig. 6.17. These effects are ignored in the beam model that predicts a two-dimensional motion.

The bending moments $M_{11}$ at the center point of each panel are shown in fig. 6.18. Pronounced discrepancies are observed between the shell and beam models because the latter does not take into account the bending-twisting coupling behavior in the panels. Note the much higher frequency content predicted by the shell model. This is due to the twisting and transverse bending of the panels that occur at much higher frequencies than those associated with longitudinal bending. Significant twisting $M_{12}$ and transverse bending $M_{22}$ moments develop in the panels, as depicted in fig. 6.19; of course, these twisting moments 


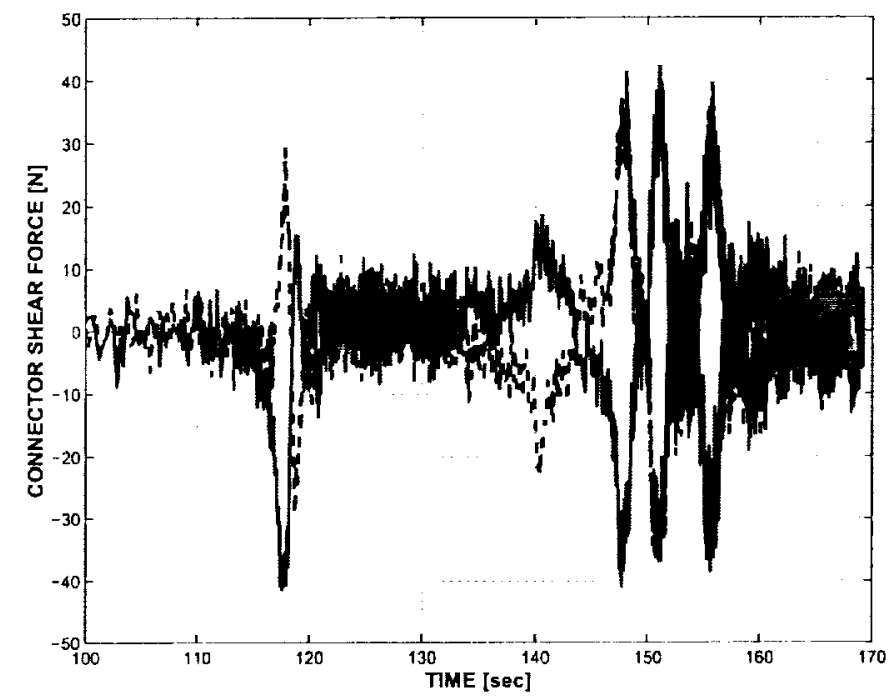

Fig. 6.17. Transverse shear forces in the connector beams between Panels 1 and 2. Ream $C_{1} A_{2}$ : solid line; beam $D_{1} B_{2}$ : dashed line.
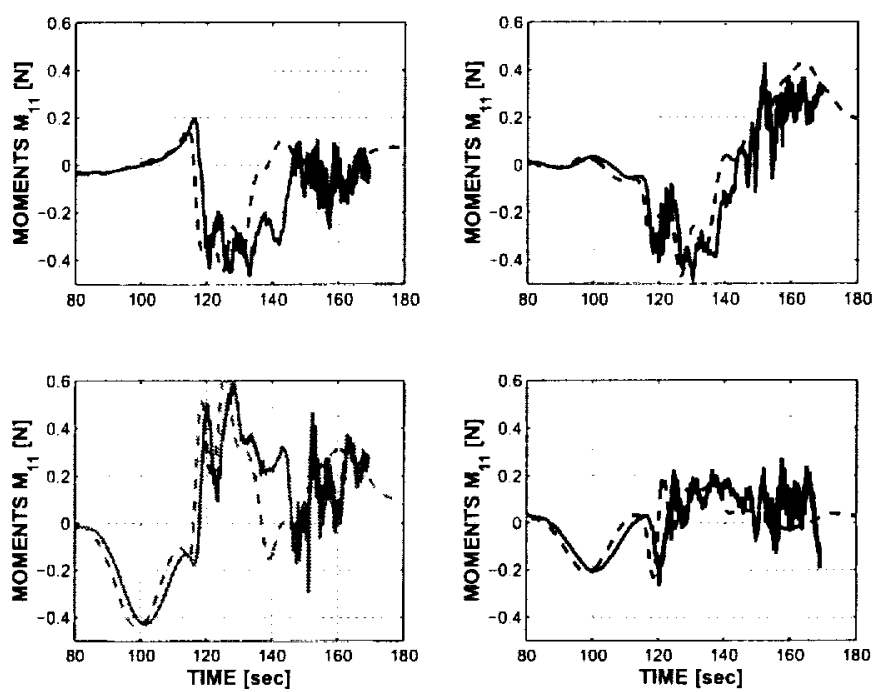

FIG. 6.18. Mid-span bending moments $M_{11}$ in the panels. Panel 1: top-left, Panel 2: top-right, Panel 3: bottom-left, Panel 4: bottom-right figure. Shell model: solid line; beam model: dashed line.

vanish in the beam model. Note that the transverse bending moment $M_{22}$ is of the same order of magnitude as the longitudinal bending moment $M_{11}$, confirming the plate-like nature of the deformation in each panel, an effect ignored by the beam model.

7. Conclusions. In this work, a new geometric integration procedure was developed for the simulation of multibody system dynamics involving shells. The proposed scheme is "aware" of the qualitative features of the underlying partial differential equations. In particular, it evolves on the special manifold defined by two-parameter rotation fields, on the manifold defined by the presence of holonomic and non-holonomic constraints imposed by the mechanical joints and on the manifold of constant total linear and angular momenta.

In contrast with the classical energy preserving approaches, the method presented here lets the system 

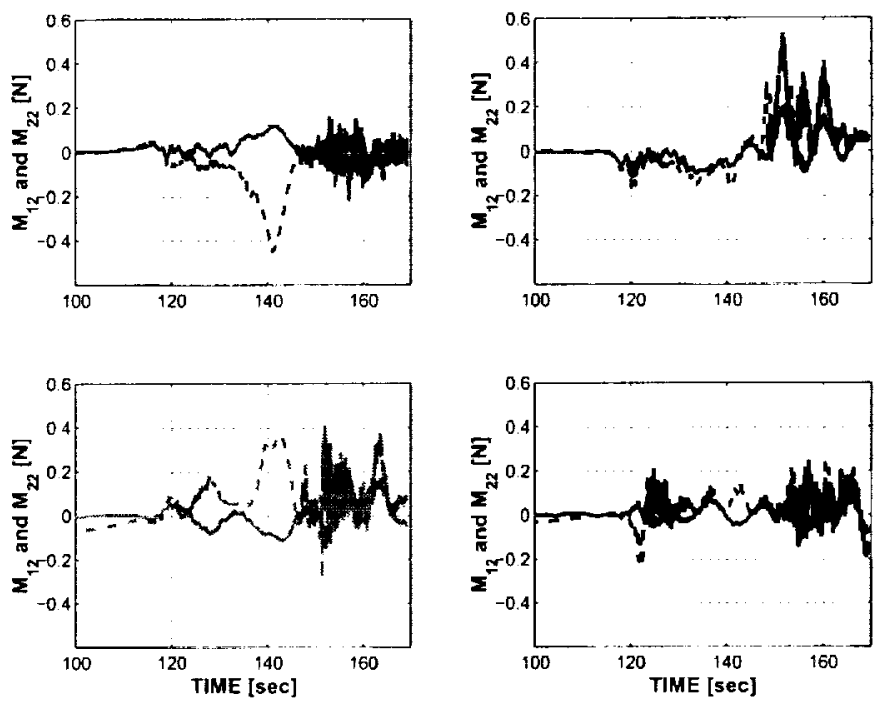

FIG. 6.19. Mid-span twisting $\left(M_{12}\right)$ and bending $\left(M_{22}\right)$ moments in the panels. Panel $1:$ top-left, Panel 2: top-right, Panel 3: bottom-left, Panel 4: bottom-right figure.

drift away from the level of constant energy in a controlled manner. This achieves two important goals. First, a bound is placed on the total mechanical energy of the system at the discrete solution level, therefore achieving nonlinear unconditional stability. Second, the energy decay provides high frequency numerical dissipation. The proposed integrator can be used with arbitrary spatial discretizations, for example based on finite element or finite volume techniques. The shear locking phenomenon was controlled using the mixed interpolation of tensorial components approach.

The proposed shell model was developed within the framework of a multibody dynamics analysis procedure that includes rigid bodies, beams, and various types of joints. The constraint forces are discretized so that the work they perform vanishes exactly at the discrete level. A shell revolute joint that connect shells to other elements of the model was developed. The efficiency and robustness of the proposed approach were demonstrated with specific numerical examples.

\section{REFERENCES}

[1] K. BathF, and E. Dvorkin, A four-node plate bending element based on Mindlin/ Reissner plate theory and a mixed interpolation, International Journal for Numerical Methods in Engineering, 21 (1985), pp. 367-383.

[2] — A formulation of general shell elements - The use mixed interpolation of tensorial components, International Journal for Numerical Methods in Engineering, 22 (1986), pp. 697-722.

[3] O. BavChat, Computational schemes for flexible, nonlinear multi-body systems, Multibody System Dynamics, 2 (1998), pp. 169-225.

[4] O. BauchaU AND C. BotTasso, On the design of energy preserving and decaying schemes for flexible, nonlinear multi-body systems, Computer Methods in Applied Mechanics and Engineering, 169 (1999), pp. 6179.

[5] - Robust integration schemes for flexible multibody systems, Computer Methods in Applied Mechanics and Engineering, (2000). Under revicw. 
[6] O. Bauchay, G. Damitano, and N. ThFron, Numerical integration of nonlinear elastic multi-body systems, International Journal for Numerical Methods in Engincering, 38 (1995), pp. 27272751.

[7] O. BAvChaU AND T. Joo, Computational schemes for nonlinear elasto-dynamics, International Journal for Numerical Methods in Engineering, 45 (1999), pp. 693719.

[8] O. Bauchau and N. Throm, Energy decaying scheme for non-linear beam models, Computer Methods in Applied Mechanics and Engineering, 134 (1996), pp. 3756.

[9] _ Energy decaying schemes for nonlinear elastic multi-body systems, Computers and Structures, 59 (1996), pp. 317331.

[10] M. Borri, C. Bottasso, and L. Trainfidi, A novel momentum-preserving energy-decaying algorithm for finite element multibody procedures, in Proceedings of Computational Aspects of Nonlinear Structural Systems with Large Rigid Body Motion, NATO Advanced Research Workshop, Pultusk, Poland, July 2-7, 2000.

[11] M. Borri, L. Trainfi.t, and C. Bottasso, On representations and parameterizations of motion, Multibody Systems Dynamics, 4 (2000), pp. 129-193.

[12] C. Bottasso, O. Banchad, AND J. Chol, An energy decaying scheme for nonlinear dynamics of shells, Computer Methods in Applied Mechanics and Engineering, (2000). Under review.

[13] C. BotTasso AND M. BORRT, Energy preserving/decaying schemes for non-linear beam dynamics using the helicoidal approximation, Computer Methods in Applied Mechanics and Engineering, 143 (1997), pp. 393415.

[14] _ Integrating finite rotations, Computer Methods in Applied Mechanics and Enginecring, 164 (1998), pp. 307331.

[15] C. Bottasso, M. BORRI, AND L. TRainflit, Integration of elastic multibody systems using invariant preserving/dissipating schemes. Part I formulation \& Part II numerical schemes and applications., Computer Methods in Applied Mechanics and Engineering, (2000). Accepted, to appear.

[16] M. BUCAIFM AND K. BATHF, Higher-order MITC general shell elements, International Journal for Numerical Methods in Engineering, 36 (1993), pp. 3729-3754.

[17] C. BUDD AND A. ISFRt.fs, Geometric integration: Numerical solution of differential equations on manifolds, Philosophical Transactions of the Royal Society of Iondon Series A-Mathematical Physical and Engineering Sciences, 357 (1999), pp. 945-956.

[18] J. ChUng AND G. HutBerT, A time integration algorithm for structural dynamics with improved numerical dissipation: The generalized- $\alpha$ method, Journal of Applied Mechanics, 122 (1995), pp. 254 266.

[19] H. Hither, T. HughFs, AND R. TAYIOR, Improved numerical dissipation for time integration algorithms in structural dynamics, Earthquake Engincering and Structural Dynamics, 5 (1977), pp. $282-$ 292.

[20] T. Kanf and D. Levinson, Dynamics: Theory and Applications, McGraw-Hill, Inc., New York, 1985.

[21] J. Simo and N. TARnOW, The discrete energy-momentum method. Conserving algorithms for nonlinear dynamics, ZAMP, 43 (1992), pp. 757-792.

$[22]$ _ A new energy and momentum conserving algorithm for the nonlinear dynamics of shells, International Journal for Numerical Methods in Engineering, 37 (1994), pp. 2527-2549.

[23] J. Simo, N. TaRnow, and M. Dobi.ARF, Non-linear dynnmics of three-dimensional rods: Exact energy and momentum conserving algorithms, International Journal of Numerical Methods in Engineering, 38 (1995), pp. 14311473 
[24] J. SimO AND K. WONG, Unconditionally stable algorithms for rigid body dynamics that exactly preserve energy and momentum, International Journal for Numerical Methods in Engineering: 31 (1991), pp. 1952.

\section{Appendix A. Rodrigues Parameters.}

A common representation of finite rotations [20] is in terms of Rodrigues parameters $\underline{r}=2 \underline{k} \tan \phi / 2$, where $\phi$ is the magnitude of the finite rotation and $\underline{k}$ the components of the unit vector about which it takes place. The following notation is introduced $r_{0}=\cos ^{2} \phi / 2=1 /\left(1+\underline{r}^{T} \underline{r} / 4\right)$, and the finite rotation tensor $R$ then writes

$$
R(\underline{r})=I+r_{0} \widetilde{r}+\frac{r_{0}}{2} \widetilde{r} \widetilde{r}
$$

The following decomposition of the rotation tensor is extensively used in this work

$$
R=\left(I+\frac{\tilde{r}}{2}\right)\left(I+\frac{\tilde{r}}{2}\right)^{-T}=\left(I+\frac{\tilde{r}}{2}\right)^{-T}\left(I+\frac{\tilde{r}}{2}\right) ; \quad\left(I+\frac{\tilde{r}}{2}\right)^{-T}=\frac{R+I}{2} .
$$

\section{Appendix B. Orientation of a Unit Director.}

Consider a unit vector $\underline{i}_{3}$, called a director, that rotates to a final orientation $\underline{e}_{3}$. For convenience, this director is considered to be the third unit vector of a triad $\mathcal{S}$ defined by $\underline{i}_{1}, \underline{i}_{2}, \underline{i}_{3}$, rotating to a triad $\mathcal{S}^{*}$ with orientation $\underline{e}_{1}, \underline{e}_{2}, \underline{e}_{3}$. The relationship between these two triads is $\underline{e}_{\alpha}=R \underline{i}_{\alpha}$, where $R$ is an orthogonal rotation tensor. If one solely focuses on the director, this rotation tensor is not uniquely defined, as any rotation about the director leaves its orientation unchanged. A virtual change in the director orientation is

$$
\delta \underline{e}_{3}=\tilde{e}_{3}^{T} \underline{\delta \psi},
$$

where $\delta \psi$ is the virtual rotation vector, $\widetilde{\delta \psi}=\delta R R^{T}$.

The components of the virtual change in director orientation measured in $\mathcal{S}^{*}$ become

$$
R^{T} \delta \underline{e}_{3}=R^{T} \widetilde{e}_{3}^{T} \underline{\delta \psi}=\widetilde{i}_{3}^{T} R^{T} \underline{\delta \psi}=\widetilde{i}_{3}^{T} \underline{\delta \psi^{*}}=\left|\begin{array}{c}
-\delta \psi_{2}^{*} \\
\delta \psi_{1}^{*} \\
0
\end{array}\right|
$$

where $\delta \psi^{*}$ are the components of the virtual rotation vector in $\mathcal{S}^{*}$. This relationship clearly demonstrates that arbitrary values of $\delta \psi_{3}^{*}$, corresponding to virtual rotations of the director about its own orientation, will not affect virtual changes in the director orientation, and hence, setting $\delta \psi_{3}^{*}=0$ is a valid choice. The following notation is adopted

$$
\underline{\delta \psi^{*}}=\underline{i}_{1} \delta \alpha_{1}^{*}+\underline{i}_{2} \delta \alpha_{2}^{*}=b \underline{\delta \alpha^{*}} ; \quad b=\left[\underline{i}_{1}, \underline{i}_{2}\right] .
$$

$\underline{\delta \alpha^{*}}$ is a $2 \times 1$, "two parameter" virtual rotation vector. It follows that $\underline{\delta \psi}=R \underline{\delta \psi^{*}}=R b \underline{\delta \alpha^{*}}$, and hence

$$
\delta \underline{e}_{\alpha}=R \widetilde{i}_{\alpha}^{T} b \underline{\delta \alpha}^{*} .
$$

If Rodrigues parameters are used to parameterize $R$, an equivalent expression can be obtained for finite changes in director orientation with the help of eq. (A.2)

$$
\underline{e}_{\alpha f}-\underline{e}_{\alpha i}=R_{m} \vec{i}_{\alpha}^{T} b \underline{s}^{*}=Q_{m} \underline{s}^{*} ; \quad \underline{r}^{*}=b \underline{s}^{*},
$$

where $\underline{r}^{*}$ are the Rodrigues parameter measured in $\mathcal{S}^{*}$, and $\underline{s}^{*}$ the corresponding "two parameter" incremental rotation vector. 


\begin{tabular}{|c|c|c|}
\hline \multicolumn{2}{|c|}{ REPORT DOCUMENTATION PAGE } & $\begin{array}{l}\text { Form Approved } \\
\text { OMB No. 0704-0188 }\end{array}$ \\
\hline \multicolumn{3}{|c|}{ 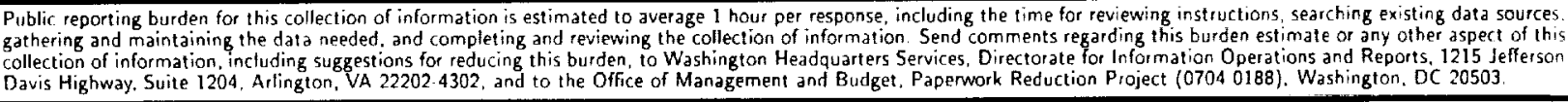 } \\
\hline 1. AGENCY USE ONLY(Leave blank) & $\begin{array}{l}\text { 2. REPORT DATE } \\
\text { November } 2000\end{array}$ & $\begin{array}{l}\text { 3. REPORT TYPE AND DATES COVERED } \\
\text { Contractor Report }\end{array}$ \\
\hline \multicolumn{2}{|c|}{$\begin{array}{l}\text { 4. TITLE AND SUBTITLE } \\
\text { On the modeling of shells in multibody dynamics }\end{array}$} & \multirow[t]{2}{*}{$\begin{array}{l}\text { 5. FUNDING NUMBERS } \\
\text { C NAS1-97046 } \\
\text { WU 505-90-52-01 }\end{array}$} \\
\hline \multicolumn{2}{|c|}{$\begin{array}{l}\text { 6. AUTHOR(S) } \\
\text { Olivier A. Banchau, Jou-Young Choi, and Carlo L. Bottasso }\end{array}$} & \\
\hline \multicolumn{2}{|c|}{$\begin{array}{l}\text { 7. PERFORMING ORGANIZATION NAME(S) AND ADDRESS(ES) } \\
\text { ICASE } \\
\text { Mail Stop 132C } \\
\text { NASA Langley Research Center } \\
\text { Hampton, VA } 23681-2199\end{array}$} & $\begin{array}{l}\text { 8. PERFORMING ORGANIZATION } \\
\text { REPORT NUMBER } \\
\text { ICASE Report No. } 2000-42\end{array}$ \\
\hline \multicolumn{2}{|c|}{$\begin{array}{l}\text { 9. SPONSORING/MONITORING AGENCY NAME(S) AND ADDRESS(ES) } \\
\text { National Aeronautics and Space Administration } \\
\text { Langley Research Center } \\
\text { Hampton, VA 23681-2199 }\end{array}$} & $\begin{array}{l}\text { 10. SPONSORING/MONITORING } \\
\text { AGENCY REPORT NUMBER } \\
\text { NASA/CR-2000-210556 } \\
\text { ICASE Report No. } 2000-42\end{array}$ \\
\hline \multicolumn{3}{|c|}{$\begin{array}{l}\text { 11. SUPPLEMENTARY NOTES } \\
\text { Langley Technical Monitor: Dennis M. Bushnell } \\
\text { Final Report } \\
\text { Submitted to Multibody Dynamics Systems. }\end{array}$} \\
\hline \multicolumn{2}{|c|}{$\begin{array}{l}\text { 12a. DISTRIBUTION/AVAILABILITY STATEMENT } \\
\text { Unclassified-Unlimited } \\
\text { Subjert Category } 64 \\
\text { Distribution: Nonstandard } \\
\text { Availability: NASA-CASI (301) 621-0390 }\end{array}$} & 12b. DISTRIBUTION CODE \\
\hline \multicolumn{3}{|c|}{$\begin{array}{l}\text { 13. ABSTRACT (Maximum } 200 \text { words) } \\
\text { Energy preserving/decaying schemes are presented for the simulation of the nonlinear multibody systems involving } \\
\text { shell components. The proposed schemes are designed to meet four specific requirements: unconditional nonlinear } \\
\text { stability of the scheme, a rigorous treatment of both geometric and material nonlinearities, exact satisfaction of the } \\
\text { constraints, and the presence of high frequency numerical dissipation. The kinematic nonlinearities associated with } \\
\text { arbitrarily large displacements and rotations of shells are treated in a rigorous manner, and the material nonlinearities } \\
\text { can be handled when the constitutive laws stem from the existcnce of a strain energy density function. The efficiency } \\
\text { and robustness of the proposed approach is illustrated with specific numerical examples that also demonstrate the } \\
\text { need for integration schemes possessing high frequency numerical dissipation. }\end{array}$} \\
\hline
\end{tabular}

